\title{
Wrong sign bottom Yukawa coupling in low energy supersymmetry
}

\author{
Nina M. Coyle, ${ }^{1}$ Bing Li, ${ }^{1}$ and Carlos E. M. Wagner ${ }^{1,2,3}$ \\ ${ }^{1}$ Physics Department and Enrico Fermi Institute, University of Chicago, Chicago, Illinois 60637, USA \\ ${ }^{2}$ Kavli Institute for Cosmological Physics, University of Chicago, Chicago, Illinois 60637, USA \\ ${ }^{3}$ High Energy Physics Division, Argonne National Laboratory, Argonne, Illinois 60439, USA
}

(Received 26 March 2018; published 15 June 2018)

\begin{abstract}
The study of the Higgs boson properties is one of the most relevant activities in current particle physics. In particular, the Higgs boson couplings to third generation fermions is an important test of the mechanism of mass generation. In spite of their impact on the production and decay properties of the Higgs boson, the values of these couplings are still uncertain and, in models of new physics, they can differ in magnitude as well as in sign with respect to the Standard Model case. In this article, we study the possibility of a wrong sign bottom-quark Yukawa coupling within the framework of the minimal (MSSM) and next-to-minimal (NMSSM) supersymmetric Standard Model. We find that a wrong sign regime is not possible in the MSSM without violating experimental constraints, and we numerically examine the parameter values which give a wrong sign bottom Yukawa within the NMSSM. Possible experimental tests are also discussed, including novel decays of the heavy $C P$-even and $C P$-odd Higgs fields that may be probed in the near future and that may lead to an explanation of some intriguing di-boson signatures observed at the ATLAS experiment.
\end{abstract}

DOI: 10.1103/PhysRevD.97.115028

\section{INTRODUCTION}

The Higgs discovery $[1,2]$ has led to the confirmation of the Standard Model as the proper effective theory at the weak scale. No new particle has been seen at the LHC, implying that new physics at the weak scale should be weakly interacting or that strongly interacting particles, if present, should lead to signatures involving soft decay products or in channels with large irreducible backgrounds. Searches for new physics under these conditions should be complemented by precision measurements of the properties of the Standard Model particles as well as rare processes rates.

Although the gauge sector of the Standard Model has been tested with high precision, the Higgs sector properties are still greatly unknown. The signal strength of different production and decay channels is in overall agreement with the Standard Model, but the errors are still large, and the coupling of the Higgs with third generation quarks and leptons is subject to big uncertainties. Indeed, while the central value of the production rate of the Higgs in association with top quarks is currently somewhat larger than the SM value [3-5], the central value of the production rate of the Higgs decaying into bottom quarks and

Published by the American Physical Society under the terms of the Creative Commons Attribution 4.0 International license. Further distribution of this work must maintain attribution to the author(s) and the published article's title, journal citation, and DOI. Funded by SCOAP ${ }^{3}$. produced in association with heavy gauge bosons, which seemed to be low at run I, is now in rough agreement with the SM prediction $[3,6,7]$. Since the errors in these determinations are still quite large, it is interesting to consider the possibility that the couplings of the Higgs to top and bottom quarks differ from the SM values due to new physics effects.

In this article, we shall consider the possibility that not only the magnitude but also the sign of the Higgs coupling to bottom quarks differ from the Standard Model predictions. This is an intriguing possibility that could be realized in the simplest two-Higgs-doublet extension of the Standard Model [8]. Such a region of parameters has been invoked recently also in models that lead to large rates of lepton flavor violating decays of the Higgs bosons $h \rightarrow \tau \mu$ [9] and on theories of flavor at the weak scale [10]. In this article we study the possible realization of this scenario within the minimal supersymmetric extensions of the SM, namely the MSSM [11-14] and the NMSSM [15].

Low energy supersymmetry [16] leads to the stability of the weak scale under the large radiative effects induced by possible heavy particles, like the ones associated with a hypothetical grand unified theory (GUT). It also leads to the radiative breaking of the electroweak symmetry, induced by corrections associated with the superpartners of the third generation quarks. The low energy theory contains at least two Higgs doublets and therefore the coupling of the Higgs to bottom quarks may be affected by mixing between the different $C P$-even Higgs bosons in the theory. 
Large negative variation of the bottom quark coupling to the SM-like Higgs boson may be obtained in the MSSM at large values of $t_{\beta}$, the standard ratio of the Higgs vacuum expectation values, and values of the heaviest $C P$-even Higgs boson masses not far above the weak scale [8]. This region of parameter space, however, is strongly restricted by searches for Higgs bosons decaying into $\tau$-lepton pairs $[17,18]$, which makes the realization of this scenario difficult. As we shall show in this article, the scenario is more easily realized in the NMSSM, although the necessary values of the coupling $\lambda$ of the singlet to the doublet Higgs superfields are larger than the ones leading to perturbative consistency of the theory up to the GUT scale.

This article is organized as follows. In Sec. II we analyze the possibility of a wrong sign Yukawa coupling within twoHiggs-doublet models. In Sec. III we study the possible realization of this idea within the MSSM and the NMSSM. After pointing out the difficulties of its realization in the MSSM, in Sec. IV we present an analytical and numerical analysis of this question within the NMSSM. In Secs. V and VI we study the experimental probes of this scenario. We reserve Sec. VIII for our conclusions.

\section{WRONG SIGN YUKAWA IN TYPE II TWO-HIGGS-DOUBLET MODELS}

The tree-level couplings of the lightest Higgs boson to gauge bosons and fermions in type II 2HDM Higgs are listed as below,

$$
\begin{gathered}
g_{h V V}=g_{h V V}^{\mathrm{SM}} s_{\beta-\alpha}, \\
g_{h t \bar{t}}=\frac{m_{t}}{v} \frac{c_{\alpha}}{s_{\beta}} \equiv \frac{m_{t}}{v}\left(s_{\beta-\alpha}+c_{\beta-\alpha} t_{\beta}^{-1}\right), \\
g_{h b \bar{b}}=-\frac{m_{b}}{v} \frac{s_{\alpha}}{c_{\beta}} \equiv \frac{m_{b}}{v}\left(s_{\beta-\alpha}-c_{\beta-\alpha} t_{\beta}\right),
\end{gathered}
$$

where $s_{\alpha}\left(s_{\beta}\right)=\sin \alpha(\sin \beta), c_{\alpha}\left(c_{\beta}\right)=\cos \alpha(\cos \beta), t_{\beta}=$ $\tan \beta$ and $s_{\beta-\alpha}\left(c_{\beta-\alpha}\right)=\sin (\beta-\alpha)\left(c_{\beta-\alpha}\right)$. As we can see from Eq. (2), for the gauge boson couplings to be SMlike, we need $s_{\beta-\alpha} \approx 1$. In this case, for moderate values of $t_{\beta}$, the Higgs coupling to top quarks or other up-type fermions becomes SM-like due the $t_{\beta}$ suppression of the second term on the right-hand side of Eq. (2). However, for the Higgs to b-quark coupling, Eq. (3), a wrong sign could arise without changing the Higgs decay width and branching ratio when $g_{h b \bar{b}} / g_{h b \bar{b}}^{\mathrm{SM}} \simeq-1$. This could be achieved with minor changes of the Higgs couplings to top quarks and weak gauge bosons for sizable values of $t_{\beta}$ and $[19,20]$

$$
t_{\beta} c_{\beta-\alpha} \approx 2
$$

This is in contrast with the condition $t_{\beta} c_{\beta-\alpha} \simeq 0$ that ensures a SM-like coupling of the bottom quark to the Higgs boson.

The scalar potential of the most general two-Higgsdoublet extension of the SM may be written as

$$
\begin{aligned}
V= & m_{11}^{2} \Phi_{1}^{\dagger} \Phi_{1}+m_{22}^{2} \Phi_{2}^{\dagger} \Phi_{2}-m_{12}^{2}\left(\Phi_{1}^{\dagger} \Phi_{2}+\text { H.c. }\right) \\
& +\frac{1}{2} \lambda_{1}\left(\Phi_{1}^{\dagger} \Phi_{1}\right)^{2}+\frac{1}{2} \lambda_{2}\left(\Phi_{2}^{\dagger} \Phi_{2}\right)^{2}+\lambda_{3}\left(\Phi_{1}^{\dagger} \Phi_{1}\right)\left(\Phi_{2}^{\dagger} \Phi_{2}\right) \\
& +\lambda_{4}\left(\Phi_{1}^{\dagger} \Phi_{2}\right)\left(\Phi_{2}^{\dagger} \Phi_{1}\right)+\left\{\frac{1}{2} \lambda_{5}\left(\Phi_{1}^{\dagger} \Phi_{2}\right)^{2}+\left[\lambda_{6}\left(\Phi_{1}^{\dagger} \Phi_{1}\right)\right.\right. \\
& \left.\left.+\lambda_{7}\left(\Phi_{2}^{\dagger} \Phi_{2}\right)\right] \Phi_{1}^{\dagger} \Phi_{2}+\text { H.c. }\right\} .
\end{aligned}
$$

After converting to the Higgs basis [21,22], the Higgs potential above could be rewritten as

$$
\begin{aligned}
\mathcal{V} & \supset \cdots+\frac{1}{2} Z_{1}\left(H_{1}^{\dagger} H_{1}\right)^{2}+\cdots \\
& +\left[Z_{5}\left(H_{1}^{\dagger} H_{2}\right)^{2}+Z_{6}\left(H_{1}^{\dagger} H_{1}\right) H_{1}^{\dagger} H_{2}+\text { H.c. }\right]+\cdots,
\end{aligned}
$$

where we have only retained those terms relevant for the following discussion and the new couplings $Z_{i}^{\prime} s$ are associated with previous $\lambda_{i}^{\prime} s$ by the following relations [23-25]:

$Z_{1} \equiv \lambda_{1} c_{\beta}^{4}+\lambda_{2} s_{\beta}^{4}+\frac{1}{2}\left(\lambda_{3}+\lambda_{4}+\lambda_{5}\right) s_{2 \beta}^{2}+2 s_{2 \beta}\left[c_{\beta}^{2} \lambda_{6}+s_{\beta}^{2} \lambda_{7}\right]$,

$Z_{5} \equiv \frac{1}{4} s_{2 \beta}^{2}\left[\lambda_{1}+\lambda_{2}-2\left(\lambda_{3}+\lambda_{4}+\lambda_{5}\right)\right]+\lambda_{5}-s_{2 \beta} c_{2 \beta}\left(\lambda_{6}-\lambda_{7}\right)$,

$$
\begin{aligned}
Z_{6} \equiv & -\frac{1}{2} s_{2 \beta}\left[\lambda_{1} c_{\beta}^{2}-\lambda_{2} s_{\beta}^{2}-\left(\lambda_{3}+\lambda_{4}+\lambda_{5}\right) c_{2 \beta}\right] \\
& +c_{\beta} c_{3 \beta} \lambda_{6}+s_{\beta} s_{3 \beta} \lambda_{7} .
\end{aligned}
$$

The $C P$-even Higgs mixing angle in this basis is identified with $\beta-\alpha$. Consequently, we have [23,25-27]

$$
c_{\beta-\alpha}=\frac{-Z_{6} v^{2}}{\sqrt{\left(m_{H}^{2}-m_{h}^{2}\right)\left(m_{H}^{2}-Z_{1} v^{2}\right)}} .
$$

As stressed before, since the observed Higgs boson has SM-like properties, $s_{\beta-\alpha} \simeq 1$, in order to fulfill the requirement to obtain a negative sign of the bottom Yukawa coupling, Eq. (4), sizable values of $t_{\beta}$ are required. For large values of $t_{\beta}, s_{\beta} \simeq 1, c_{\beta} \simeq 1 / t_{\beta}$ and $s_{2 \beta} \simeq 2 / t_{\beta}$. Since $Z_{1} v^{2} \simeq m_{h}^{2}$, the denominator becomes approximately $m_{H}^{2}-m_{h}^{2}$. From the relation of $Z_{6}$ to the quartic couplings $\lambda_{i}$ we obtain that, ignoring subdominant terms in $1 / t_{\beta}$, an 
inversion of the sign of the Yukawa coupling leads to the following condition:

$$
\left[\left(\lambda_{3}+\lambda_{4}+\lambda_{5}\right)-\lambda_{2}+\lambda_{7} t_{\beta}\right] v^{2} \simeq 2\left(m_{H}^{2}-m_{h}^{2}\right),
$$

or, equivalently,

$$
\left[\left(\lambda_{3}+\lambda_{4}+\lambda_{5}\right)+\lambda_{7} t_{\beta}\right] v^{2} \simeq 2 m_{H}^{2}-m_{h}^{2},
$$

where we have used the fact that, at large values of $t_{\beta}$, $m_{h}^{2} \simeq \lambda_{2} v^{2}$. Hence, considering perturbative values of the quartic couplings $\lambda_{i}$, it is straightforward to see that, unless $\lambda_{7} \simeq \mathcal{O}(1)$, the values of $m_{H}$ must be of order of a few hundred $\mathrm{GeV}$.

The relation between the charged and neutral Higgs masses is given by

$$
m_{H^{ \pm}}^{2}=m_{A}^{2}+\frac{v^{2}}{2}\left(\lambda_{5}-\lambda_{4}\right)
$$

and hence large values of $\lambda_{4}$ and $\lambda_{5}$ may induce a large splitting between the charged and the $C P$-odd Higgs boson masses.

\section{WRONG SIGN YUKAWA COUPLINGS IN THE MSSM AND THE NMSSM}

\section{A. MSSM and minimal NMSSM}

The tree-level Higgs sector of the MSSM is a type-II 2HDM and consists of two Higgs doublets with quartic couplings which are related to the squares of the weak gauge couplings. Since supersymmetry imposes concrete values for the quartic couplings $\lambda_{i}$ it is interesting to check whether the wrong sign Yukawa coupling could arise in the frame of the MSSM without conflicting with other Higgs phenomenology. For this, one has to take into account the relevant radiative corrections arising from the interaction of the Higgs field with the third generation fermions and their scalar superpartners. In the MSSM, it is usually argued that a SM-like neutral Higgs boson could only be obtained in two distinct scenarios, i.e., the decoupling limit [23-44] and the alignment limit $[23,24,44,45]$. The decoupling limit happens when $m_{h} \ll m_{H}$, while the alignment limit arises when one of the $C P$-even Higgs bosons, when expressed as a linear combination of the real parts of the two neutral Higgs fields, lies in the same direction in the two-Higgsdoublet field space as neutral Higgs vacuum expectation values. This alignment does not in general depend on the masses of the nonstandard Higgs bosons. The region of parameters under investigation requires a nonvanishing value of $c_{\beta-\alpha}$ and therefore a departure from the alignment limit. Hence, some departures from the SM behavior of the lightest Higgs are expected.

In the MSSM, it is not difficult to work out an approximate expression for $Z_{6}$ at the one-loop level.
Taking into account that the most relevant radiative corrections may be absorbed in the definition of the Higgs mass at large values of $t_{\beta}$, one gets

$$
\begin{aligned}
\lambda_{2} & \simeq \frac{m_{h}^{2}}{v^{2}} \\
\lambda_{3} & \simeq \frac{1}{4}\left(g_{2}^{2}-g_{1}^{2}\right) \\
\lambda_{4} & \simeq-\frac{1}{2} g_{2}^{2} \\
\lambda_{7} & \simeq \frac{3 h_{t}^{4}}{16 \pi^{2}} \frac{\mu}{M_{S}}\left(\frac{A_{t}^{3}}{6 M_{S}^{3}}-\frac{A_{t}}{M_{S}}\right) \\
m_{t} & \simeq h_{t} \frac{v}{\sqrt{2}},
\end{aligned}
$$

where $m_{t}$ is the top quark mass, $M_{S}$ is the stop mass scale, $A_{t}$ is the trilinear stop-Higgs coupling and $\mu$ is the Higgsino mass parameter (for a more complete expression, see Refs. [28-30]). Taking into account Eq. (10), we get the following estimate [25]:

$$
t_{\beta} c_{\beta-\alpha} \simeq \frac{1}{m_{H}^{2}-m_{h}^{2}}\left[-m_{h}^{2}-m_{Z}^{2}+\frac{3 m_{t}^{4}}{4 \pi^{2} v^{2}} \frac{\mu}{M_{S}}\left(\frac{A_{t}^{3}}{6 M_{S}^{3}}-\frac{A_{t}}{M_{S}}\right) t_{\beta}\right],
$$

where the first two terms inside the square bracket come from $\left(\lambda_{3}+\lambda_{4}+\lambda_{5}-\lambda_{2}\right) v^{2}$, while the last term comes from the radiatively induced $\lambda_{7} v^{2} t_{\beta}$ contribution.

If we want $t_{\beta} c_{\beta-\alpha}$ to be as large as 2 , it is clear that we need the third term in the square bracket to be quite large. Unfortunately, this will lead to an unacceptably large value of $t_{\beta}$, which pushes the Yukawa coupling to third generation down-type fermions to large values that are restricted by heavy Higgs searches [17,18]. In order to see that, let us recall the fact that stability of the Higgs potential demands that $\left|A_{t}\right|$ and $|\mu|$ should both be smaller than $3 M_{S}$ [31]. Under this constraint, the maximum of the expression $\frac{\mu}{M_{S}}\left(\frac{A_{t}^{3}}{6 M_{S}^{3}}-\frac{A_{t}}{M_{S}}\right)$ is 4.5 , which is achieved for $A_{t} / M_{S}=3$ and $\mu / M_{S}=3$. When normalized in terms of the square of the Higgs mass, the coefficient $\frac{3 m_{t}^{4}}{4 \pi^{2} v^{2}}$ is about $m_{h}^{2} / 16$, which is very small compared to the first two positive terms in the square brackets. Thus, for $t_{\beta} c_{\beta-\alpha}$ to reach the target value $2, t_{\beta}$ needs to be very large. More specifically, for $m_{H} \approx 250 \mathrm{GeV}$, one requires values of $t_{\beta} \approx 30$, while for $m_{H} \approx 500 \mathrm{GeV}, t_{\beta} \approx 120$. These values of $m_{H}$ and $t_{\beta}$ are excluded by heavy Higgs searches at the LHC $[17,18]$. One could avoid these constraints for larger values of the heavy Higgs mass, larger than $1 \mathrm{TeV}$. However, for $m_{H} \approx 1 \mathrm{TeV}$ one requires $t_{\beta} \approx 500$, and it is difficult to keep the perturbative consistency of the theory at such large values of $t_{\beta}$. 
In order to address the question of perturbative consistency of the MSSM at large values of $t_{\beta}$, we should stress that in supersymmetric models there are relevant radiatively generated couplings of the $H_{u}$ Higgs boson to the bottom quarks, which imply a departure from the simple type II 2HDM. These modifications are particularly important for large values of the Higgsino mass parameter $\mu$ and lead to a modification of $\kappa_{b}$ [46]:

$$
\kappa_{b}=\frac{g_{h b b}}{g_{h b b}^{\mathrm{SM}}}=-\frac{s_{\alpha}}{c_{\beta}}\left[1-\frac{\Delta_{b}}{1+\Delta_{b}}\left(1+\frac{1}{t_{\alpha} t_{\beta}}\right)\right],
$$

where $\Delta_{b}$ is given by [47-49]

$\Delta_{b} \simeq\left(\frac{2 \alpha_{3}}{3 \pi} M_{3} \mu I\left(m_{\tilde{b}_{1}}, m_{\tilde{b}_{2}}, M_{3}\right)+\frac{h_{t}^{2}}{(4 \pi)^{2}} A_{t} \mu I\left(m_{\tilde{t}_{1}}, m_{\tilde{t}_{2}}, \mu\right)\right) t_{\beta}$

and the function $I(a, b, c)$ is given by

$$
\begin{aligned}
& I(a, b, c) \\
& \quad=\frac{a^{2} b^{2} \ln \left(a^{2} / b^{2}\right)+b^{2} c^{2} \ln \left(b^{2} / c^{2}\right)+c^{2} a^{2} \ln \left(c^{2} / a^{2}\right)}{\left(a^{2}-c^{2}\right)\left(a^{2}-b^{2}\right)\left(b^{2}-c^{2}\right)}
\end{aligned}
$$

and $M_{3}, m_{\tilde{b}_{i}}, m_{\tilde{t}_{i}}$ are the gluino, sbottom and stop mass eigenvalues.

There are similar corrections to the tau coupling, but they are governed by weak coupling effects and are therefore less significant. The above corrections imply a difference between $\kappa_{b}$ and $\kappa_{\tau}$ and therefore have relevant phenomenological consequences for sizable values of $t_{\beta}$. In particular, in the region of parameters under investigation, $\kappa_{\tau}$ tends to differ from $\kappa_{b}$ by a few tens of percent.

Moreover, the coupling of the heavy MSSM-like Higgs bosons to bottom quarks becomes

$$
g_{H b \bar{b}} \simeq g_{A b \bar{b}} \simeq h_{b} \simeq \frac{m_{b} t_{\beta}}{v\left(1+\Delta_{b}\right)} .
$$

These corrections must be in general considered when studying the production and decay of the heavy $C P$-even and $C P$-odd Higgs bosons and lead to some moderate modification of these rates with respect to the ones expected in the type II $2 \mathrm{HDM}$.

At very large values of $t_{\beta}$, the bottom Yukawa coupling $h_{b}$ and the $\tau$ Yukawa coupling $h_{\tau} \simeq m_{\tau} t_{\beta} / v$ become large. For positive values of $\Delta_{b}$, the increase of the bottom Yukawa coupling is slower than what is expected using the tree-level relations and hence perturbative consistency can be kept for larger values of $t_{\beta}$. However, for sizable $\Delta_{b}$, it can be easily shown that the condition to invert the sign of the bottom Yukawa coupling becomes

$$
t_{\beta} c_{\beta-\alpha}=2\left(1+\Delta_{b}\right) .
$$

Therefore, for very large values of $t_{\beta}$ there is a tension between maintaining the perturbative consistency of the theory, which depends on $h_{b}$ and as shown in Eq. (19) is more easily fulfilled for positive $\Delta_{b}$, and the fulfillment of Eqs. (15) and (20). Thus we reach the conclusion that within the MSSM the current LHC bounds make it very difficult to invert the sign of the Higgs coupling to bottom quarks while keeping the perturbative consistency of the theory at low energies.

Next let us turn to the next-to-minimal supersymmetric extension of the SM (NMSSM) [15], with only an extra singlet superfield added on top of the MSSM. The $C P$-even singlet will mix with the two neutral $C P$-even Higgs bosons. We consider first the simpler case when the superpotential is scale invariant and thus the complete Lagrangian would have an accidental $\mathbb{Z}_{3}$ symmetry. The superpotential is given by

$$
\begin{aligned}
W= & \lambda \hat{S} \hat{H}_{u} \cdot \hat{H}_{d}+\frac{\kappa}{3} \hat{S}^{3}+h_{u} \hat{Q} \cdot \hat{H}_{u} \hat{U}_{R}^{c}+h_{d} \hat{H}_{d} \cdot \hat{Q} \hat{D}_{R}^{c} \\
& +h_{\ell} \hat{H}_{d} \cdot \hat{L} \hat{E}_{R}^{c},
\end{aligned}
$$

where $\hat{S}, \widehat{H_{u}}, \widehat{H_{d}}$ denote the singlet and doublet Higgs superfields, and $\hat{Q}, \hat{D}_{R}, \hat{U}_{R}$ are the quark superfields, while $\hat{L}, \hat{E}_{R}$ are the lepton superfields, $h_{i}$ are the Yukawa couplings and $\lambda$ and $\kappa$ are both dimensionless couplings. Note that in this case, $\mu$ is an effective mass parameter generated by the vev of the singlet, $\mu_{\text {eff }}=\lambda s$; we will use $\mu$ in the following discussions to refer to $\mu_{\text {eff }}$. Observe that the fields $H_{u}$ and $H_{d}$ have opposite hypercharge. These fields can be related to the fields $\Phi_{1}$ and $\Phi_{2}$ introduced before by the relations

$$
H_{d}^{i}=\epsilon_{i j} \Phi_{1}^{j *} \quad H_{u}^{i}=\Phi_{2}^{i}
$$

and therefore

$$
H_{u} H_{d}=-\Phi_{2}^{\dagger} \Phi_{1} .
$$

The most significant change in the NMSSM would be that at tree level,

$$
\delta \lambda_{4}=\lambda^{2}
$$

and therefore there is an extra correction proportional to $\frac{1}{2} \lambda^{2} v^{2} s_{2 \beta}^{2}$ in the $M_{11}^{2}$ term of the Higgs basis. This term is relevant since it can lift up the upper limit of the lightest Higgs mass at tree level [34], thus making it possible to reach $125 \mathrm{GeV}$ without the large quantum corrections needed in the MSSM [32,33,35-39]. What is more important in this case is that it can modify the $Z_{6}$ term introduced earlier in the MSSM case and release the strong tension between $t_{\beta}$ and 
$M_{A}$ present in the MSSM to make $c_{\beta-\alpha} t_{\beta}=2$ feasible. In the NMSSM, considering heavy singlets, it is straightforward to get the expression for $Z_{6}$ at moderate or large values of $t_{\beta}$, including only the stop loop corrections, namely [50],

$Z_{6} v^{2} \approx \frac{1}{t_{\beta}}\left[m_{h}^{2}+m_{Z}^{2}-\lambda^{2} v^{2}\right]+\frac{3 v^{2} h_{t}^{4} \mu X_{t}}{16 \pi^{2} M_{S}^{2}}\left(1-\frac{X_{t}^{2}}{6 M_{S}^{2}}\right)$,

where $X_{t}=A_{t}-\mu / t_{\beta}$, which leads to

$$
\begin{aligned}
t_{\beta} c_{\beta-\alpha} \approx & \frac{-1}{m_{H}^{2}-m_{h}^{2}}\left[\left(m_{h}^{2}+m_{Z}^{2}-\lambda^{2} v^{2}\right)\right. \\
& \left.+\frac{3 m_{t}^{4} A_{t} \mu t_{\beta}}{4 \pi^{2} v^{2} M_{S}^{2}}\left(1-\frac{A_{t}^{2}}{6 M_{S}^{2}}\right)\right] .
\end{aligned}
$$

Compared with Eq. (15), we have an extra $-\lambda^{2} v^{2}$ term in the parentheses, which comes from $\delta \lambda_{4}$, Eq. (24), and tends to push $t_{\beta} c_{\beta-\alpha}$ towards positive values, making it promising to get $t_{\beta} c_{\beta-\alpha}=2$ with smaller values of $t_{\beta}$. However, for that purpose we need $\lambda$ to be of order 1 . For $\lambda$ or $\kappa$ of order 1 , one can no longer neglect the chargino, neutralino and Higgs loop contributions when evaluating the Higgs mass and couplings. Although approximate analytical expressions exist in this case, the formulas become complicated and beyond some approximate formulas we will present in the next section, we will mostly base our results in a numerical analysis with full quantum corrections up to two-loop level, which are necessary to select the proper region of parameter space leading to the inversion of the bottom coupling. Moreover, large $\lambda$ could lead to a Landau pole problem at energies lower than the grand unification scale. We reserve a discussion of this issue to the Appendix.

\section{B. Moderate values of $\boldsymbol{t}_{\boldsymbol{\beta}}$}

As we discussed before, the MSSM is highly constrained for the large values of $t_{\beta}$ necessary to achieve an inversion of the bottom couplings. These constraints tend to translate into the NMSSM since for very large values of $t_{\beta}$ the decay of the MSSM-like $C P$-even and $C P$-odd Higgs bosons into bottom quarks and $\tau$ leptons remain relevant. In the NMSSM, however, for sufficiently large values of $\lambda$, an inversion of the sign of the SM-like Higgs boson coupling to bottom quarks may be achieved at moderate values of $t_{\beta} \simeq 5-10$. For this range of values of $t_{\beta}$ the constraints from direct searches for heavy Higgs bosons become weaker, but some of the approximations performed before cease to be valid. In particular, the effects of the mixing between the $C P$-even Higgs bosons become relevant and cannot be dismissed. Due to these mixing effects, and ignoring the mixing with singlets, we obtain

$$
m_{h}^{2} \simeq Z_{1} v^{2}-\frac{Z_{6}^{2} v^{4}}{m_{H}^{2}}
$$

or, equivalently

$$
m_{h}^{2} \simeq Z_{1} v^{2}-c_{\beta-\alpha}^{2} m_{H}^{2} \simeq \lambda_{2} v^{2}-\frac{4 m_{H}^{2}}{t_{\beta}^{2}} .
$$

For very large values of $t_{\beta}$ the last term may be safely ignored. However, for moderate values of $t_{\beta}$ this term cannot be ignored and tends to push the mass of the Higgs boson to values that are below the experimentally observed value. Mixing with the singlets only worsens this situation. In order to address this problem, a departure from the simple $\mathbb{Z}_{3}$ invariant NMSSM is necessary.

\section{NMSSM with singlet tadpole terms}

As discussed above, we are interested in the inversion of the sign of the coupling of the bottom quark to the Higgs in the simplest NMSSM case, with sizable values of $\lambda$ and moderate values of $t_{\beta}$. This simple framework tends to lead to problems in the $C P$-even Higgs sector, since as we discussed in the previous section, the lightest $C P$-even Higgs boson mass is generically pushed to values below the experimentally observed ones due to large mixing effects. A possible solution to this problem is to include a nonzero singlet tadpole term $\xi_{S}$ to the potential

$$
\Delta V=\xi_{S} S+\text { H.c. }
$$

This term, which could be a result of the supersymmetry breaking mechanism at high scales [40-42], serves to break the $\mathbb{Z}_{3}$ symmetry explicitly and get rid of unwanted domain walls. For values of $\mu$ of the order of the weak scale and $\lambda$ couplings of order one, a sizable value of $\left|\xi_{S}\right|$ leads to large values of the singlet mass. Ignoring other terms that become subdominant for large values of $\left|\xi_{S}\right|$, one obtains

$$
\langle S\rangle=\frac{\mu}{\lambda} \simeq-\frac{\xi_{S}}{m_{S}^{2}}
$$

or, equivalently

$$
m_{S}^{2} \simeq-\frac{\lambda \xi_{S}}{\mu}
$$

A sizable $\left|\xi_{S}\right|$ could keep the singlet decoupled from the two neutral Higgs bosons, reducing the problem to an approximate $2 \times 2$ Higgs mixing one, with low energy quartic couplings that are modified by terms proportional to powers of the couplings $\lambda$ and $\kappa$. For moderate values of $\xi_{S}$, the decoupling effects may affect the low energy theory in a relevant way. We shall discuss these effects in more detail below. 
An additional consequence of large values of $\xi_{S}$ is that the singlet mass may become much larger than the mass of the singlino. In this case, the quartic coupling of $H_{u}$ has sizable corrections produced by $\lambda^{4}$ loop contributions from singlets and singlinos. The correction to $\lambda_{2}$ from these contributions is given by

$$
\delta \lambda_{2} \simeq \frac{\lambda^{4}}{16 \pi^{2}} \ln \left(\frac{m_{S}^{2}}{\mu^{2}}\right) \simeq \frac{\lambda^{4}}{16 \pi^{2}} \ln \left(\left|\frac{\lambda \xi_{S}}{\mu^{3}}\right|\right),
$$

where we have used the expression for $m_{S}^{2}$ given in Eq. (31). It is therefore clear that for values of $|\mu|$ of the order of the weak scale, large values of $\xi_{S}$ result in large positive corrections to $\lambda_{2}$. These corrections can compensate the negative contributions to the Higgs mass induced by mixing effects and constrain the allowable values of $\xi_{S}$ via the experimental constraints on the lightest $C P$-even Higgs mass, which will be examined in more specificity in the next section.

A further possible modification to the NMSSM is the inclusion of a similar tadpole term in the superpotential, namely beyond the trilinear terms associated with the Yukawa, $\lambda$ and $\kappa$ couplings, one may add a tadpole term of the form [15]

$$
\delta W=\xi_{F} S,
$$

where $\xi_{F}$ is a dimension 2 parameter. One action of such a term, as we shall discuss, is to modify the spectral relationships between the neutral and charged Higgs bosons. In our initial analysis, we first set $\xi_{F}=0$; however, we shall discuss the impact of this term in later examinations of pseudoscalar decays in Sec. VI.

The decoupling of the singlet induces corrections to $\lambda_{4}$ and $\lambda_{5}$, and a sizable correction to the quartic coupling $\lambda_{7}$. This can be seen by ignoring subdominant terms and reducing the singlet-dependent terms in the scalar potential to

$$
\begin{aligned}
& \left(m_{S}^{2}+\lambda^{2}\left|H_{u}\right|^{2}\right)|S|^{2}+\left[S\left(\lambda A_{\lambda} H_{u} H_{d}+\xi_{S}\right)+\text { H.c. }\right] \\
& \quad+\left|\xi_{F}+\lambda H_{u} H_{d}+\kappa S^{2}\right|^{2},
\end{aligned}
$$

where we shall assume that, due to the effect of the tadpole terms, $m_{S}^{2}$ is much larger than $\lambda^{2} H_{u}^{2} \simeq \lambda^{2} v^{2}$. From Eq. (34), and ignoring small corrections induced by the vacuum expectation values of the singlet and doublet fields, we can see that the masses of the $C P$-even and $C P$-odd singlet eigenstates are approximately given by

$$
m_{h_{S}}^{2}=m_{S}^{2}+2 \xi_{F} \kappa, \quad m_{A_{S}}^{2}=m_{S}^{2}-2 \xi_{F} \kappa,
$$

respectively. Now, one can integrate out the singlets, replacing the singlet fields by their equation of motion. This is roughly

$$
\begin{aligned}
& \operatorname{Re}(S) \simeq-\frac{\lambda A_{\lambda}\left(H_{u} H_{d}+\text { H.c. }\right)+\xi_{S}}{2 m_{h_{S}}^{2}}, \\
& \operatorname{Im}(S) \simeq-i \frac{\lambda A_{\lambda}\left(H_{u} H_{d}-\text { H.c. }\right)}{2 m_{A_{S}}^{2}} .
\end{aligned}
$$

Replacing this expression into the original Lagrangian density, Eq. (34), one obtains contributions to $\lambda_{4}, \lambda_{5}$, and $\lambda_{7}$ given by

$$
\begin{aligned}
\delta \lambda_{4} \simeq & -\lambda^{2}\left(\frac{A_{\lambda}^{2}}{2 m_{h_{S}}^{2}}+\frac{A_{\lambda}^{2}}{2 m_{A_{S}}^{2}}\right)+2 \lambda^{2} \kappa \frac{\xi_{S} A_{\lambda}}{m_{h_{S}}^{2}}\left(\frac{1}{m_{h_{S}}^{2}}-\frac{1}{m_{A_{S}}^{2}}\right) \\
& +\frac{\xi_{F} A_{\lambda}^{2} \kappa \lambda^{2}}{2}\left(\frac{1}{m_{h_{S}}^{4}}-\frac{1}{m_{A_{S}}^{4}}\right)+\frac{\kappa^{2} \lambda^{2} A_{\lambda}^{2} \xi_{S}^{2}}{m_{h_{S}}^{4}}\left(\frac{3}{m_{h_{S}}^{4}}+\frac{1}{m_{A_{S}}^{4}}\right) \\
\delta \lambda_{5} \simeq & -\lambda^{2}\left(\frac{A_{\lambda}^{2}}{2 m_{h_{S}}^{2}}-\frac{A_{\lambda}^{2}}{2 m_{A_{S}}^{2}}\right)+2 \lambda^{2} \kappa \frac{\xi_{S} A_{\lambda}}{m_{h_{S}}^{2}}\left(\frac{1}{m_{h_{S}}^{2}}+\frac{1}{m_{A_{S}}^{2}}\right) \\
& +\frac{\xi_{F} A_{\lambda}^{2} \kappa \lambda^{2}}{2}\left(\frac{1}{m_{h_{S}}^{4}}+\frac{1}{m_{A_{S}}^{4}}\right)+\frac{\kappa^{2} \lambda^{2} A_{\lambda}^{2} \xi_{S}^{2}}{m_{h_{S}}^{4}}\left(\frac{3}{m_{h_{S}}^{4}}-\frac{1}{m_{A_{S}}^{4}}\right) \\
\delta \lambda_{7} \simeq & -\lambda^{3} \frac{\xi_{S} A_{\lambda}}{m_{h_{S}}^{4}} .
\end{aligned}
$$

The value of $\mu A_{\lambda}$ is related to the $C P$-odd Higgs spectrum by the relation

$$
\left[\mu\left(A_{\lambda}+\frac{\kappa}{\lambda} \mu\right)+\lambda \xi_{F}\right] t_{\beta} \simeq M_{A}^{2}
$$

and therefore for fixed $M_{A}$ and moderate values of $\kappa$, sizable negative values of $\xi_{F}$ result in large positive values of $\mu A_{\lambda}$. Hence, for values of $\mu$ at the weak scale, the presence of negative $\xi_{F}$ can lead to sizable values of $A_{\lambda}$ and therefore to large corrections to $\lambda_{7}$. Such large corrections may induce a modification of the value of $c_{\beta-\alpha}$, which including only the dominant terms becomes

$$
\begin{aligned}
t_{\beta} c_{\beta-\alpha} \approx & \frac{1}{m_{H}^{2}-m_{h}^{2}}\left[\left(\lambda^{2}\left(1-\frac{A_{\lambda}^{2}}{m_{h_{S}}^{2}}\right) v^{2}-\lambda_{2} v^{2}-M_{Z}^{2}\right)\right. \\
& \left.+\frac{3 m_{t}^{4} A_{t} \mu t_{\beta}}{4 \pi^{2} v^{2} M_{S}^{2}}\left(\frac{A_{t}^{2}}{6 M_{S}^{2}}-1\right)-\lambda^{3} v^{2} \frac{\xi_{S} A_{\lambda} t_{\beta}}{m_{h_{S}}^{4}}\right] .
\end{aligned}
$$

Hence, the reduction of the $\lambda^{2}$ contribution due to sizable values of $A_{\lambda}$ may be compensated by the explicit $\xi_{S}$ dependence appearing in the last term of Eq. (39).

Moreover, including the above corrections to $\lambda_{4}$ and $\lambda_{5}$ modifies the difference between the charged and neutral $C P$-odd Higgs boson masses, Eq. (13), 


$$
\begin{aligned}
\left(\lambda_{5}-\lambda_{4}\right) \frac{v^{2}}{2} \simeq & M_{W}^{2}+\left\{\left(\frac{A_{\lambda}^{2}}{m_{A_{S}}^{2}}-1\right)\right. \\
& \left.+\kappa\left[\frac{4 \xi_{S} A_{\lambda}}{m_{A_{S}}^{2} m_{h_{S}}^{2}}+\left(\xi_{F}-\frac{2 \kappa \xi_{S}^{2}}{m_{h_{S}}^{4}}\right) \frac{A_{\lambda}^{2}}{m_{A_{S}}^{4}}\right]\right\} \frac{\lambda^{2} v^{2}}{2} .
\end{aligned}
$$

Hence for sizable $A_{\lambda}$, the splitting between the $C P$-odd and the charged Higgs mass induced by the large values of $\lambda$, Eq. (13), may be reduced by the effects associated with the singlet decoupling. These observations will be important in examining the constraints from precision measurements and the decay mode $A_{1} \rightarrow h Z$.

\section{NMSSM RESULTS: FULL ANALYSIS}

In this section, we conducted a numerical search for a wrong sign Yukawa coupling within the NMSSM model using NMSSMToOLS [51], which includes the most relevant one and two-loop radiative corrections to the Higgs mass matrix elements. In this calculation, we scanned over six independent parameters: $t_{\beta}, M_{A}, \mu, \lambda$, $\kappa$, and $\xi_{S}$. We fixed the gaugino and third generation scalar mass parameters to values that are not constrained by present experimental bounds and that contribute to the obtention of a proper SM-like Higgs mass at sizable values of $t_{\beta}$. This selection is somewhat arbitrary and does not play a relevant role in the Higgs phenomenology at moderate values of $t_{\beta}$. However, as we will discuss below, it has an impact on the analysis of the flavor and dark matter constraints. In our analysis we set $A_{t}=1700 \mathrm{GeV}$, $A_{\tau}, A_{b}=1500 \mathrm{GeV}$, and the squark and slepton masses at $M_{S}=1 \mathrm{TeV}$. The weak gauginos were assumed to be heavy, $M_{1,2}=1 \mathrm{TeV}$, while the gluino mass was fixed at $M_{3}=2 \mathrm{TeV}$.

As discussed in the previous section, taking into account the strong constraints on large $t_{\beta}$ for the relatively low values of the MSSM-like $C P$-odd Higgs mass [17,18] necessary to induce a large correction to the bottom coupling, we concentrate on moderate values of $t_{\beta}$. In particular, values of $t_{\beta}$ in the range 6-10 and values of the $C P$-odd Higgs mass $m_{A}$ within the interval of 300 to $500 \mathrm{GeV}$ are preferred due to these considerations. While larger values of $m_{A}$ make it more difficult to obtain a large correction to the bottom coupling, smaller values of $m_{A}$ lead to tension with the current $C P$-even and $C P$-odd neutral Higgs searches and, for $\xi_{F}=0$, to low values of the charged Higgs mass, excluded by top-quark decay studies.

In order to identify the possible ranges of $\xi_{S}$, we first performed a scan over a wide range of values of $\xi_{S}$ and $\mu$ for some characteristic parameters of the theory leading to a variation of the coupling of the Higgs to the bottom quark. An examination of Eq. (39) indicates that to obtain $t_{\beta} c_{\beta-\alpha}=2$, one requires negative values of $\mu \times \xi_{S}$. The values of $|\mu|$ are required to be at the weak scale and lead to

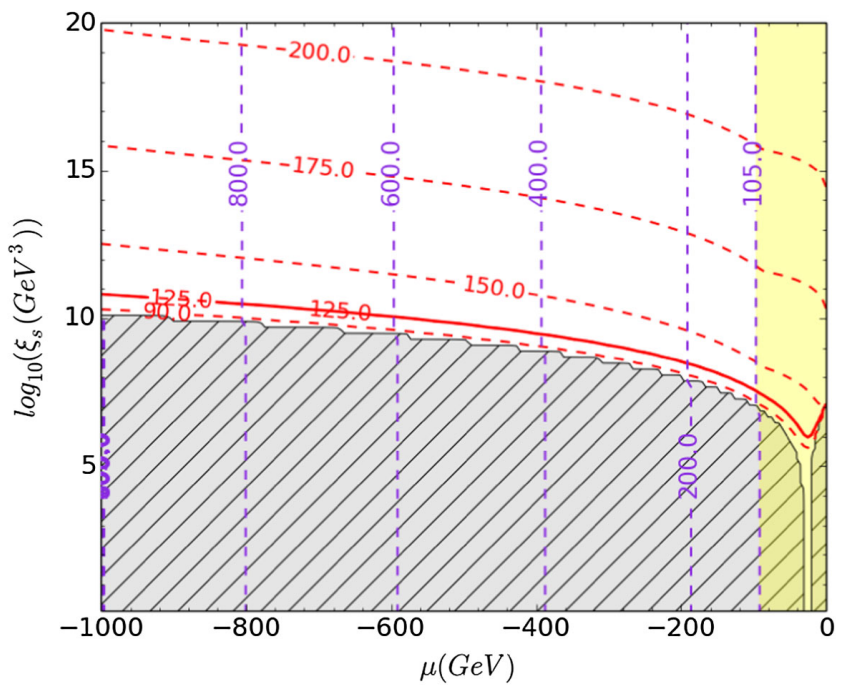

FIG. 1. In this plot, $\lambda=1.3$ and $\kappa=0.1$ are fixed, and $\mu$ and $\xi_{s}$ are varied to examine the allowable values of these parameters. The gray area is excluded for negative Higgs mass. Red contour lines indicate values of the lightest Higgs mass, with $125 \mathrm{GeV}$ represented by the solid contour. The lightest chargino mass contours are displayed in purple.

allowed values of the chargino and neutralino masses. Taking $\xi_{S}$ to be positive, we therefore scan a range for $\mu$ from -1000 to $0 \mathrm{GeV}$. All other parameters are fixed at values that favor positive values of $t_{\beta} c_{\beta-\alpha}: \lambda=1.3$, $\kappa=0.1, M_{A}=350$, and $t_{\beta}=7$. The result of this scan is shown in Fig. 1. We find that the value of the lightest $C P$ even Higgs mass $m_{1}$ does indeed increase with $\xi_{S}$, as expected from Eq. (32). The contour with $m_{1}=125 \mathrm{GeV}$ is indicated by the solid red line; the constraint of $m_{1}=125 \pm 3 \mathrm{GeV}$, which takes into account the uncertainty in the determination of the Higgs mass, therefore constrains the value of $\xi_{S}$ to be on the order of $10^{9}$ to $10^{11} \mathrm{GeV}^{3}$ for values of $|\mu|$ at the weak scale.

In the rest of this section, we examine the results of a scan to search for models which produce a wrong sign bottom Yukawa coupling. Based on the above analysis, we fix the supersymmetry mass parameters to the values given above and vary the tadpole term $\xi_{S}$ in the range of $1.25 \times 10^{10}$ and $1 \times 10^{11} \mathrm{GeV}^{3}$ and $\mu$ in the range -1300 to $-800 \mathrm{GeV}$. Beyond these ranges, the number of successful points decreases quickly due to experimental limits. For larger $\xi_{S}$ and $|\mu|$, the charged Higgs mass becomes too low; for lower $\xi_{S}$ and $|\mu|$, the range of $\xi_{S}$ and $\mu$ which passes experimental constraints and gives the correct SM-like Higgs mass becomes quite narrow, and $\kappa_{b}$ can become positive for $\mu$ close enough to 0 . We therefore do not include these ranges in the main scan. Additionally, the following parameters were varied within the ranges of $t_{\beta} \in[6,10], \quad M_{A} \in[300,400] \mathrm{GeV}, \quad \lambda \in[1.0,1.8]$, $\kappa \in[0.0,1.0]$. Parameters are randomly drawn from uniform distributions and we discard all points which give the 
wrong lightest Higgs mass or fail other collider direct experimental constraints as defined in NMSSMToOLS version 5.1.2. These constraints include constraints from heavy Higgs searches through $t \rightarrow b H^{ \pm}, H / A \rightarrow \tau \tau$, $H \rightarrow A A \rightarrow 4 l / 2 l+2 b$, and $g g F \rightarrow H / A \rightarrow \gamma \gamma$, as well as lower limits on the masses of squarks, gluino, sfermions, neutralinos, and charginos. More recent ATLAS and CMS constraints from $\phi \rightarrow \tau \tau[17,18]$ are available, where $\phi$ is an additional neutral Higgs boson, which are not included in version 5.1.2. The rate of these processes at ATLAS and CMS have been checked separately and we find that we are well within the bounds, which are of order of a few tenths of $p b$ at masses of the order of $300 \mathrm{GeV}$, due to a production cross section for $H$ or $A_{1}$ of about $1 \mathrm{pb}$ for both $\mathrm{ggF}$ and $\mathrm{b}$-associated production and a branching ratio around $2 \%$. Figure 2 shows the predicted rate in our models for ggF production of $\phi$ and subsequently $\phi$ decaying into $\tau \tau$. We did not impose any flavor or dark matter constraints, but we shall discuss these constraints in separate sections. The results of the numerical scan are shown in Fig. 3.

It is clear that the requirement of a wrong sign bottom Yukawa indeed fixes $\lambda$ to be of order 1, a reflection of the strong tree-level dependence of the bottom coupling on this parameter. Additionally, larger values of $\xi_{S}$ allow $\lambda$ to take on lower values; however, as shown in Fig. 1, $\xi_{S}$ is also restricted from the requirement of obtaining the proper Higgs mass and cannot take arbitrary large values. Therefore, it is difficult to push $\lambda$ down below order 1 using the tadpole contribution. The constraint on $\kappa$ is significantly relaxed by the large value of $\xi_{S}$, which allows $\kappa$ to take on values from 0 to 1 , with a gentle dependence on $\lambda$ for given $\xi_{S}$. Further parameters and

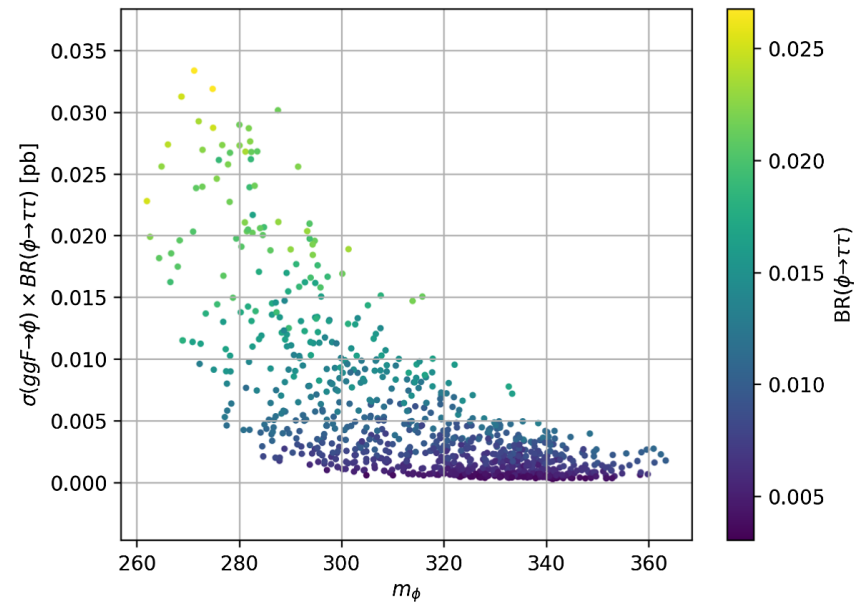

FIG. 2. Predicted rate of gluon fusion production of a heavy neutral Higgs $\phi$ decaying into $\tau \tau$, where the values are calculated including $H$ and $A_{1}$. The heavily suppressed branching ratio of $\phi \rightarrow \tau \tau$ in our models, which is discussed further below, results in a suppressed rate which is within the most recent limits. relevant outputs for five sample points which were successful are provided in Table I. It is clear from Table I that in this region of parameters $A_{\lambda} \ll m_{S}$ and therefore the effects associated with the singlet decoupling, Eq. (37), become small. As we shall see in later sections, this situation will change when we consider values of $\xi_{F} \neq 0$.

All points shown in Fig. 3 pass the experimental limits included in NMSSMTOoLS v5.1.2. Additionally, an approximately linear, $t_{\beta}$-dependent cut is applied to $m_{H^{ \pm}}$ based on the constraints provided by CMS [52]; a plot of the charged Higgs mass as a function of $m_{H}$ is shown in Fig. 4. These mass ranges allow for enhanced $H \rightarrow H^{ \pm} W^{\mp}$ and $A_{1} \rightarrow H^{ \pm} W^{\mp}$ decays, which will be discussed further in Secs. V C and VI.
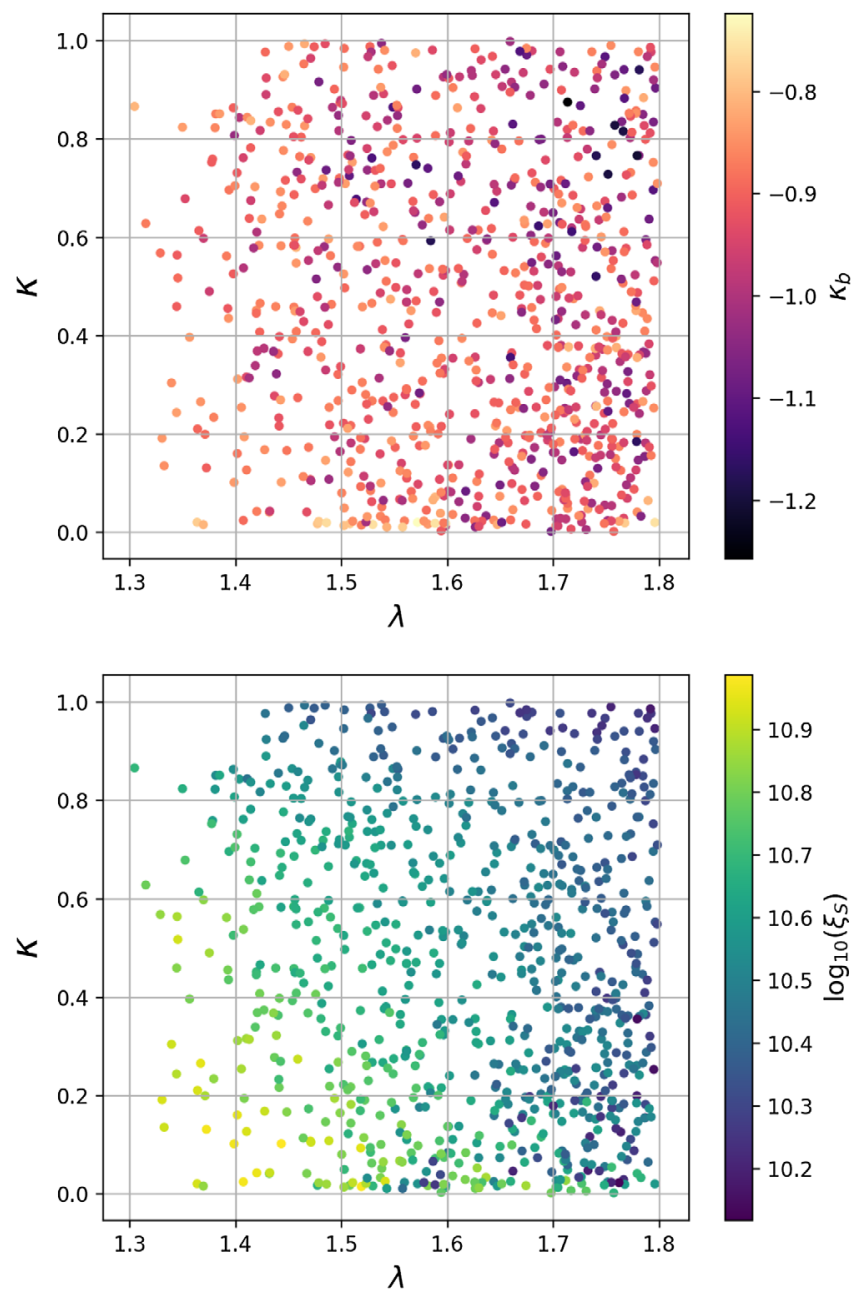

FIG. 3. Scatter plot of points that survive the $125 \mathrm{GeV}$ mass constraint and predict a wrong sign bottom Yukawa coupling. The colorbar on the upper plot shows the value of $\kappa_{b}$, which is the ratio between Higgs to $b \bar{b}$ coupling and its SM value, i.e., $g_{h b \bar{b}}^{N M S S M} / g_{h b \bar{b}}^{\mathrm{SM}}$. All points have $\kappa_{b}$ close to -1 as demanded. The lower plot shows the relationship between the values of $\lambda, \kappa$, and the tadpole contribution. 
TABLE I. Typical parameters found by NMSSMTOOLS that gave negative Higgs to $b \bar{b}$ couplings.

\begin{tabular}{|c|c|c|c|c|c|c|c|c|c|c|c|c|c|}
\hline No. & $t_{\beta}$ & $M_{A}$ & $\mu$ & $\lambda$ & $\kappa$ & $A_{\lambda}$ & $\xi_{S}$ & $\kappa_{b}$ & $\mathrm{BR}(h \rightarrow b \bar{b})$ & $m_{h}$ & $m_{H}$ & $m_{H^{ \pm}}$ & $m_{S}$ \\
\hline 1 & 9.7 & 374 & -1283 & 1.41 & 0.024 & 11.0 & $9.79 \times 10^{10}$ & -0.98 & $64.9 \%$ & 123.1 & 278 & 159 & 10360 \\
\hline 2 & 8.5 & 398 & -1294 & 1.37 & 0.131 & 109.3 & $9.38 \times 10^{10}$ & -0.90 & $63.2 \%$ & 122.3 & 271 & 158 & 9973 \\
\hline 3 & 7.7 & 369 & -1190 & 1.62 & 0.063 & 31.5 & $6.27 \times 10^{10}$ & -0.98 & $58.6 \%$ & 127.1 & 310 & 158 & 9242 \\
\hline 4 & 8.5 & 362 & -1119 & 1.41 & 0.398 & 302.3 & $6.92 \times 10^{10}$ & -0.97 & $58.9 \%$ & 126.5 & 277 & 156 & 9344 \\
\hline 5 & 8.9 & 331 & -1109 & 1.37 & 0.200 & 150.4 & $7.51 \times 10^{10}$ & -0.89 & $56.3 \%$ & 125.9 & 273 & 159 & 9634 \\
\hline
\end{tabular}

\section{IMPLICATIONS FOR LHC PHYSICS}

\section{A. SM-like Higgs boson properties}

The change of sign of the bottom coupling has implications for the loop-induced coupling of the SM-like Higgs to gluons and photons and is also correlated with changes to the couplings of nonstandard Higgs doublets to third generation quarks.

In Fig. 5, we plot the values of $\kappa_{g}$ and $\kappa_{\gamma}$ against $\kappa_{b}$, where $\kappa_{i}$ is the ratio of the Higgs coupling to the particle $i$ to its value in the SM. The $h \rightarrow g g$ and $h \rightarrow \gamma \gamma$ amplitudes have contributions from bottom quark loops, and will therefore be modified within our models. Charged Higgs loops also provide a small contribution to $h \rightarrow \gamma \gamma$ within our models given the low value of $m_{H^{ \pm}}$. All solutions show values of the couplings that are within $20 \%$ of the $\mathrm{SM}$ values, which are in agreement with current experimental constraints. These results coincide with those obtained by the authors of Ref. [8]. Interestingly enough, CMS presents a global fit to the couplings of the SM-like Higgs, assuming no new physics in either the decay or in the loop-induced couplings, leading to a marginal preference towards a wrong sign bottom Yukawa coupling [53]. Although in our model there is a small contribution to the Higgs-photon coming from the charged Higgs, the CMS preference is mostly due to an apparent enhancement of the gluon fusion-induced processes compared to the SM values, which is not present in the ATLAS data

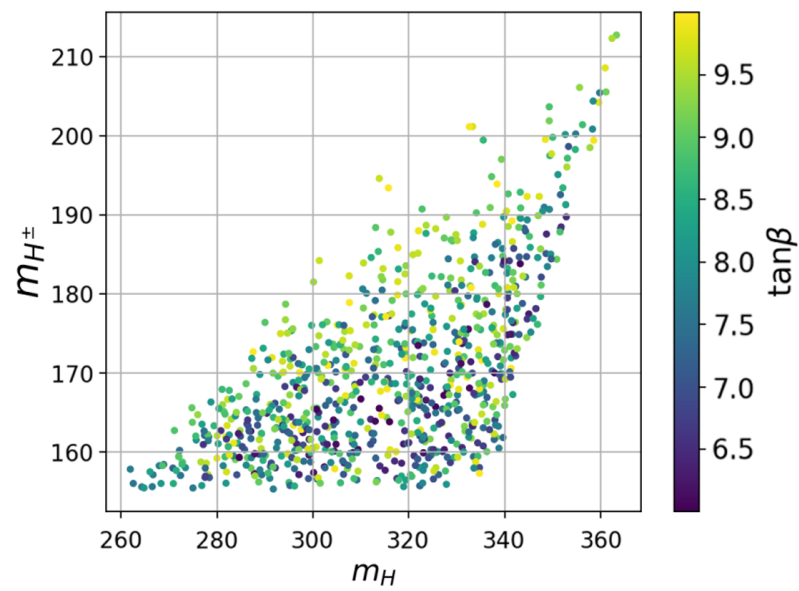

[54]. On the other hand, our values for $\kappa_{g}$ and $\kappa_{\gamma}$ are within 1 standard deviation of the most recent best-fit values from both experiments. This shows that the LHC experiments have not yet the sensitivity to distinguish between a SM-like Higgs with wrong sign bottom Yukawa couplings and the SM Higgs.

As can be seen in Fig. 5, the values of $\kappa_{g}$ for our set of points range between 1.10 and 1.13. Since we have assumed heavy supersymmetric particles, these modifications are governed by just the modifications of the bottom couplings. This is a reasonably large effect, but observing this effect at the LHC is complicated by systematic errors in the primary $g g$ fusion production cross section. Reference [55] provides expected error estimates for $\kappa_{g}$ of $6 \%-8 \%$ for an integrated luminosity of $300 \mathrm{fb}^{-1}$ and $3 \%-5 \%$ for an integrated luminosity of $3000 \mathrm{fb}^{-1}$. It is clear from these numbers that hints may become observable by the end of run 2 and the effects should be clearly resolvable by the end of run 3 .

The value of $\kappa_{\gamma}$ within our set of points ranges from approximately 0.94 to 0.98 . Estimates for LHC uncertainties in the measurement of $\kappa_{\gamma}$ are given as 5\%-7\% for $300 \mathrm{fb}^{-1}$ integrated luminosity and $2 \%-5 \%$ for $3000 \mathrm{fb}^{-1}$ integrated luminosity [55]. The measurement of $\kappa_{\gamma}$ may therefore allow an examination of the viability of the wrong sign bottom Yukawa within the NMSSM by the end of LHC run 3.

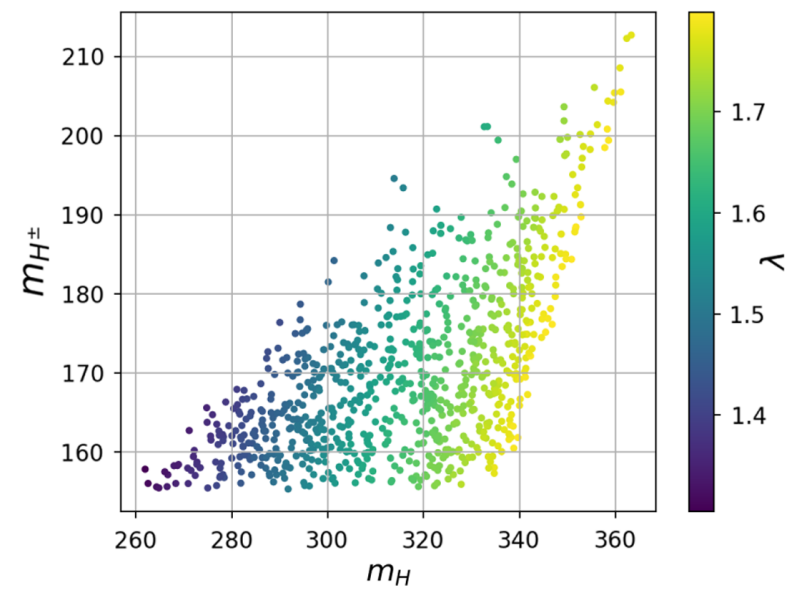

FIG. 4. Scatter plot of the charged Higgs mass $m_{H^{ \pm}}$against $m_{H}$, with the colorbar showing the value of $t_{\beta}$ (left) and $\lambda$ (right). A $t_{\beta}$-dependent mass cut on $m_{H^{ \pm}}$, with a lowest limit of $155 \mathrm{GeV}$, has been applied to satisfy experimental constraints. 

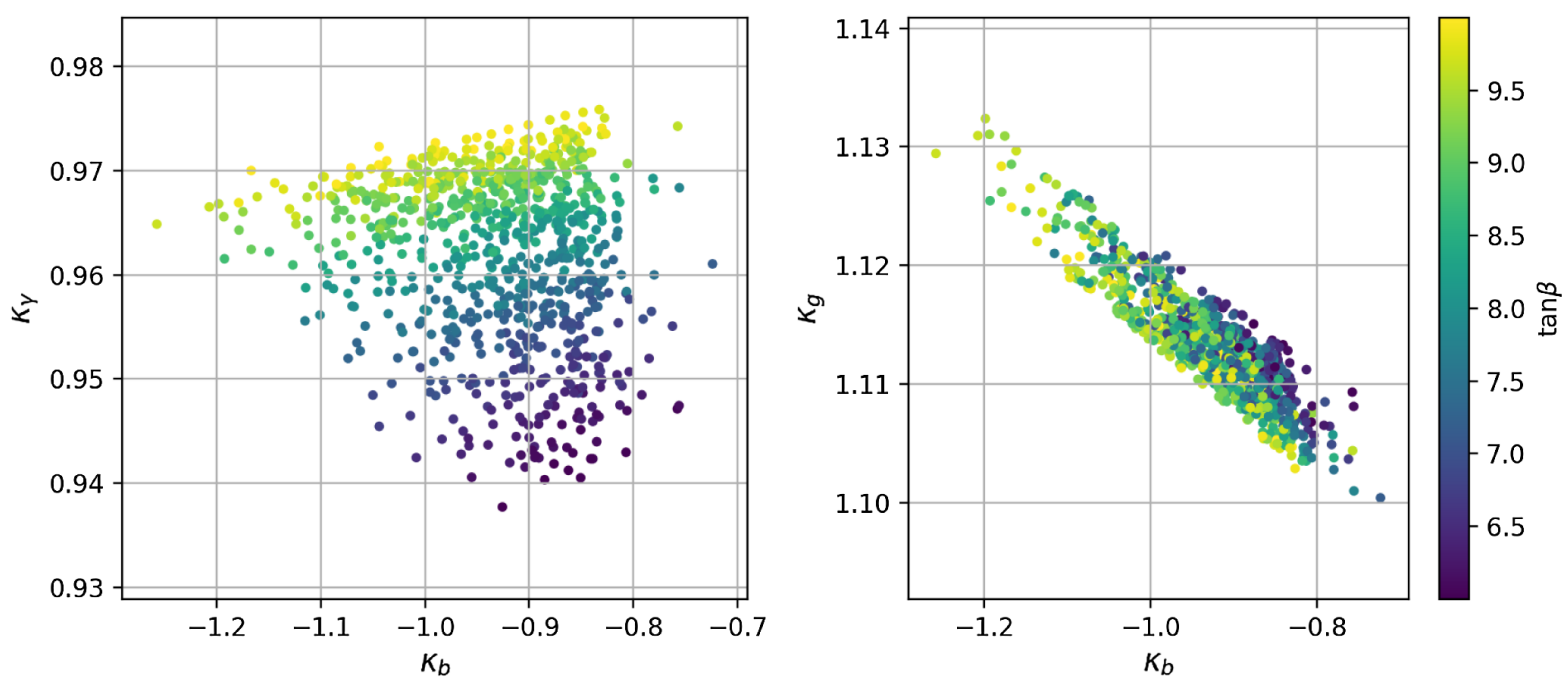

FIG. 5. Scatter plots of the couplings for the SM-like Higgs to $\gamma$ (left) and gluons (right) against $\kappa_{b}$. The colorbar indicates the value of $t_{\beta}$. We find that $\kappa_{\gamma}$ is reduced by $3 \%-6 \%$ and displays a linear dependence on $t_{\beta}$, while $\kappa_{g}$ is enhanced by approximately $10 \%-13 \%$.

Let us stress again that the above estimates of the modification of the Higgs couplings to gluons and photons have been performed under the assumption of heavy supersymmetric particles. If, eventually, charged and/or colored supersymmetric particles are detected at the LHC, their effects would have to be taken into account (see, for instance, Refs. [56-59]) in order to determine the possible effects of the inversion of the bottom coupling.

The modification of the SM-like Higgs coupling to top quarks and weak gauge bosons tend to be small in the explored region of parameters. Indeed, ignoring for simplicity the $\Delta_{b}$ corrections,

$$
\begin{aligned}
& \kappa_{W}=s_{\beta-\alpha} \simeq 1-\frac{2}{t_{\beta}^{2}} \\
& \kappa_{t}=s_{\beta-\alpha}+\frac{c_{\beta-\alpha}}{t_{\beta}} \simeq 1,
\end{aligned}
$$

where we have used the fact that $c_{\beta-\alpha} \simeq 2 / t_{\beta}$.

In Fig. 6 we show the correlation between the Higgsinduced weak diboson production cross section and the coupling of the SM-like Higgs decay into bottom quarks, normalized to the values obtained for a Higgs of the same mass in the SM. The strong correlation may be explained by the fact that the $\operatorname{BR}(h \rightarrow W W, Z Z)$ is mostly determined by the variation of the total width induced by the modification of the bottom-quark coupling to the Higgs and by the values of $\kappa_{g}^{2} \simeq 1.25$ (see Fig. 5). The outlier points which do not follow this linear relationship are associated with small values of $\kappa$, for which the SM-like Higgs boson can decay into the lightest neutralino and have therefore a nonvanishing branching ratio of decays into invisible particles.

\section{B. Radiative Higgs decay to quarkonia}

Another particular Higgs process affected by the bottom Yukawa coupling is the radiative decay of the Higgs to quarkonium, in particular to the $\Upsilon$ meson, which is composed of $b \bar{b}$. This process has also been examined within a general $2 \mathrm{HDM}$ in the wrong sign regime by [60]. Within the Standard Model, the direct and indirect Feynman diagrams have an approximate accidental cancellation, which effectively excludes this decay process at all but very high luminosities. The decay widths of $H \rightarrow \Upsilon(n S)+\gamma$ in terms of $\kappa_{b}$ are given by [61]

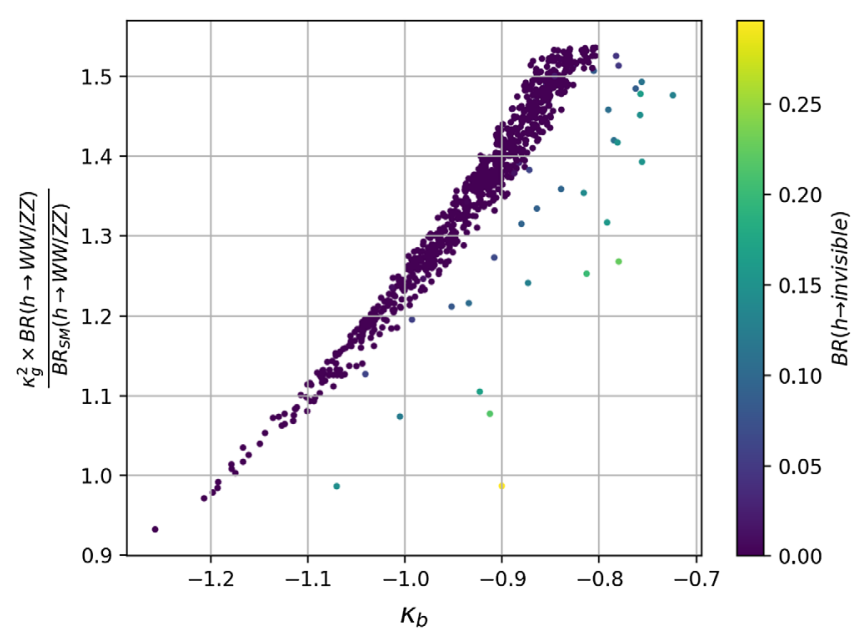

FIG. 6. Plot showing the product of $\kappa_{g}^{2}$ and the branching ratio of $h$ to $W W$ or $Z Z$ against $\kappa_{b}$. The $h \rightarrow W W / Z Z$ rates are normalized to the SM rate for the particular SM-like Higgs mass. The colorbar shows the branching ratio of the SM Higgs to neutralinos; we see that the points which do not follow the linear trend have a larger branching ratio to invisible particles. 


$$
\begin{aligned}
& \Gamma[H \rightarrow \Upsilon(1 S)+\gamma] \\
& \quad=\left|(3.33 \pm 0.03)-(3.49 \pm 0.15) \kappa_{b}\right|^{2} \times 10^{-10} \mathrm{GeV} \\
& \Gamma[H \rightarrow \Upsilon(2 S)+\gamma] \\
& \quad=\left|(2.18 \pm 0.03)-(2.48 \pm 0.11) \kappa_{b}\right|^{2} \times 10^{-10} \mathrm{GeV} \\
& \Gamma[H \rightarrow \Upsilon(3 S)+\gamma] \\
& \quad=\left|(1.83 \pm 0.02)-(2.15 \pm 0.10) \kappa_{b}\right|^{2} \times 10^{-10} \mathrm{GeV}
\end{aligned}
$$

where the first term derives from the indirect diagram and the second term, which is modified by $\kappa_{b}$, derives from the direct diagram. Note that the change in sign from $\kappa_{b}=1$ to $\kappa_{b}=-1$ gives a factor increase of between $10^{2}$ and $10^{4}$ in the decay widths. Using $\Gamma(H)=4.195_{-0.159}^{+0.164} \times 10^{-3} \mathrm{GeV}$ [62], the Higgs branching ratio to $\Upsilon(1 S, 2 S, 3 S)+\gamma$ final states for the SM are $(0.610,2.15,2.44) \times 10^{-9}$. For $\kappa_{b}=-1$, the branching ratios are $(1.11,0.518$, $0.378) \times 10^{-6}$, which are still small but significantly larger than the SM values.

The predicted number of $H \rightarrow \Upsilon(n S)+\gamma$ events at the LHC is calculated as

$$
N=\frac{\Gamma(H \rightarrow \Upsilon(n S)+\gamma)}{\Gamma(H)} \times \sigma(p+p \rightarrow H) \times \mathcal{L}_{\text {int }} .
$$

We calculate the expected number of $H \rightarrow \Upsilon(n S)+\gamma$ events for both $\kappa_{b}=1$ and $\kappa_{b}=-1$. The Higgs total cross section is taken to be $\sigma(p+p \rightarrow H)=5.57 \times$ $10^{4} \mathrm{fb}$ [62]. We examine the number of expected events by the end of LHC run 3, for which the approximate target integrated luminosity is $300 \mathrm{fb}^{-1}$. The predicted number of events is less than 1 for $\kappa_{b}=1$ and $N(\Upsilon(1 S)$, $\Upsilon(2 S), \Upsilon(3 S))=(18.5 \pm 0.7,8.65 \pm 0.36,6.31 \pm 0.26)$ for $\kappa_{b}=-1$. The number of events at the $3 \mathrm{ab}^{-1}$ highluminosity LHC is simply an order of magnitude larger than the one predicted at the end of run 3 , namely a few hundred events.

Searches for $h \rightarrow \Upsilon(n S)+\gamma$ have been performed previously for the $8 \mathrm{TeV}$ runs with approximately $20.3 \mathrm{fb}^{-1}$ of luminosity [63]. The current upper limits on the branching ratios at $95 \%$ C.L. are given for $\Upsilon(1 S, 2 S, 3 S)+\gamma$ final states as $(1.3,1.9,1.3) \times 10^{-3}$ $[63,64]$. An increase in sensitivity for these decays on the order of $10^{3}$ with respect to the one at run 1 is therefore required in order to probe the effects of a wrong sign bottom Yukawa. Therefore, despite the significant enhancement of the number of events with respect to the $\mathrm{SM}$, this process is not currently an effective method of searching for a wrong sign bottom Yukawa, and its detection will demand a significant improvement of the current analysis.

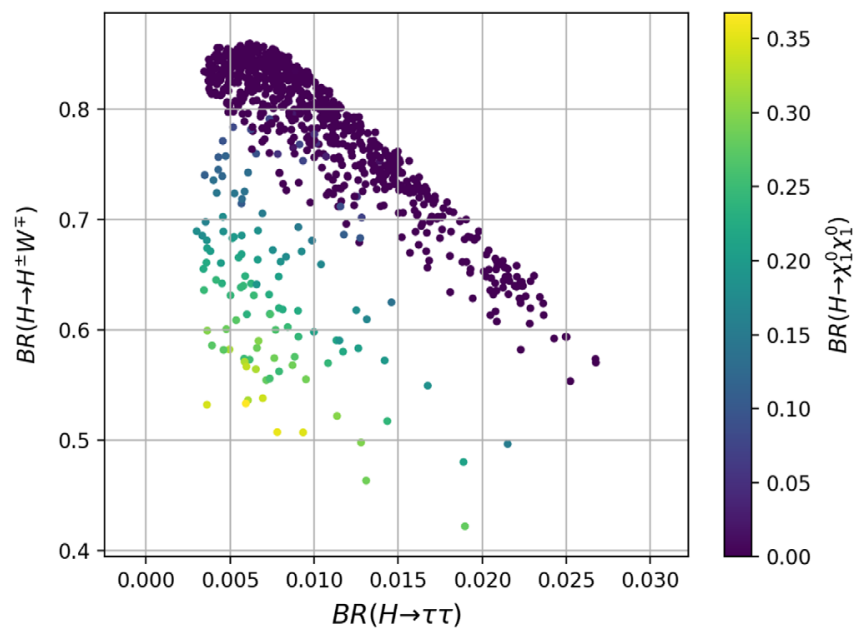

FIG. 7. Branching ratios for the decay of the heavier neutral Higgs $H$ to $H^{ \pm} W^{\mp}$ and $\tau \tau$, with the branching ratio of $H \rightarrow \chi_{1}^{0} \chi_{1}^{0}$ as the colorbar.

\section{Decay channels of the heavy neutral Higgs}

A particular characteristic of those surviving points in Table I is that they all have low charged Higgs mass. The mass difference between $H, A_{1}$, and $H^{ \pm}$allows the $H \rightarrow H^{ \pm} W^{\mp}$ channel to open up and become the dominant decay mode of the heavier neutral Higgs $H$, as well as of the lighter $C P$-odd Higgs $A_{1}$. This observation has many phenomenological consequences. On one hand, the branching ratio of $H \rightarrow \tau^{+} \tau^{-}$is suppressed even when $t_{\beta}$ is large, so that one may push $t_{\beta}$ higher than the current bounds on this channel $[17,18]$. These arguments are confirmed by Fig. 7, in which we see that all $\operatorname{BR}(H \rightarrow \tau \tau)$ values are lower than $3 \%$. For low values of $\kappa$, the singlino may become light and, as shown in Fig. 7, the invisible decay branching ratio of the heavy Higgs bosons may become relevant, and imply a further decrease of the decay into $\tau$-leptons. On the other hand, this large $\operatorname{BR}\left(H \rightarrow H^{ \pm} W^{\mp}\right)$ also means that this exotic decay channel provides a possibly interesting search channel at the LHC. Within these models, $\operatorname{BR}\left(H \rightarrow H^{ \pm} W^{\mp}\right)$ is greater than 0.4 for all models and reaches values up to 0.8. Figure 8 shows the predicted rate of $H$ decaying to $H^{ \pm} W^{\mp}$ through gluon fusion production in $p b$; we find a rate on the order of a few tenths of $p b$.

\section{Flavor constraints}

As discussed in the previous section, the presence of a light charged Higgs opens new channels for the neutral Higgs decays that can be searched at the LHC. A light charged Higgs, however, can also induce large corrections to flavor observables, in particular to the radiative decay of $B$ mesons into strange ones. In type II 2HDM's, the $b \rightarrow s \gamma$ rate is indeed highly enhanced in the presence of a charged Higgs $[65,66]$. In supersymmetric theories, however, this 


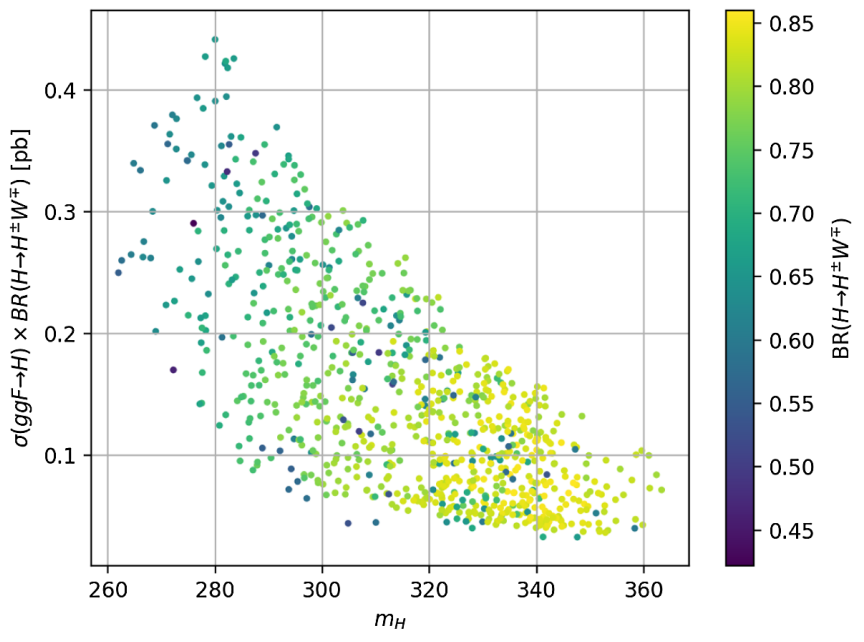

FIG. 8. Predicted rate in $p b$ of ggF-produced $H$ decaying into $H^{ \pm} W^{\mp}$.

rate depends strongly on other contributions coming from supersymmetric particles, and therefore a light charged Higgs cannot be ruled out by these considerations. On one hand, there are the contributions coming from the charginos and stops. It is indeed known that in the supersymmetric limit these cancel exactly the SM contributions to the dipole operators contributing to the $b \rightarrow s \gamma$ transition [67]. On the other hand, there are flavor violating contributions of the neutral Higgs bosons, as well as modifications of the charged Higgs couplings, coming from similar radiative corrections to the ones that contribute to $\Delta_{b}$, discussed in Sec. III B [68-71]. All these corrections are included in the NMSSMTools code we use [51]. Finally, there are contributions that are more difficult to evaluate and come from possible flavor violation in the scalar fermion sector. Those corrections are induced whenever there is a misalignment of the basis in which the quark and squark mass matrix are diagonalized, and lead to large corrections induced by gluino-squark loops [72]. These corrections are induced at the loop level even if they are not present at tree level at the supersymmetry breaking scale [73].

In view of the above, we have not considered the flavor constraints in this work. We have checked, however, that for the solutions we are presenting the flavor bounds coded in NMSSMTools have a strong dependence on the gluino mass and that small changes to $\mu$ on the order of $10 \mathrm{GeV}$ along with changes of a few hundred $\mathrm{GeV}$ of the gluino mass, from the $2 \mathrm{TeV}$ value we are considering, move models from being excluded to being in good agreement with flavor constraints. These adjustments leave the behaviors of interest in the Higgs sector unchanged. In addition, as discussed above, the low values of the charged Higgs mass depend strongly on the assumption of having just a potential tadpole for the singlet. One may push upward the value of the charged Higgs mass with the inclusion of $\xi_{F}$ in the superpotential, which decreases the mass splitting between the charged and $C P$-odd Higgs. In this case, the dependence on the gluino mass remains and flavor constraints can be satisfied with a few hundred $\mathrm{GeV}$ adjustments of $M_{3}$.

\section{HEAVY CHARGED HIGGS}

\section{A. Additional decay channels: $A_{1} \rightarrow h Z$}

As shown above, models of wrong sign Yukawa couplings have interesting phenomenological properties that go beyond the SM-like Higgs properties, and include novel decays of the heavy $C P$-even and $C P$-odd Higgs bosons that may be tested in the near future. ATLAS has recently published results which show an excess of events consistent with the production of a pseudoscalar resonance of mass about $400 \mathrm{GeV}$, produced alongside $b \bar{b}$ and decaying into $h Z$ [74]; the CMS analysis of this channel is still ongoing and has yet to be released. Although one may model this signal with a light singlet [75,76], producing such a pseudoscalar at a high enough rate through $p p \rightarrow b \bar{b} A$ production within an effective two-Higgs-doublet model requires large values of $c_{\beta-\alpha}$ and sizable values of the bottom Yukawa coupling, which are consistent with the properties of the wrong sign bottom Yukawa coupling models under study, and is therefore of interest here [19]. However, one cannot gain an $A_{1} \rightarrow h Z$ branching ratio of the necessary magnitude using the minimal models examined above due to the enhanced $A_{1} \rightarrow H^{ \pm} W^{\mp}$ decay.

In order to model the $h Z$ decay within these models, we include a nonzero value of the superpotential tadpole term $\xi_{F}$. Because $\xi_{F}$ is a dimension 2 parameter, it is therefore naturally of the order of $-10^{5}$ to $-10^{7} \mathrm{GeV}^{2}$. As noted previously from Eqs. (38) and (40), the inclusion of this term reduces the mass difference between the neutral and charged Higgs bosons and therefore suppresses the decay of the $C P$-odd Higgs boson into the charged Higgs, increasing the possible decays into $h$ and $Z$.

Introducing a nonzero $\xi_{F}$ also allows for larger values of $m_{H}$ and lower values of $\lambda$ while still satisfying $\kappa_{b} \approx-1$. As we showed explicitly in Eq. (39), the additional term arising from $\delta \lambda_{7}$ provides a positive contribution to the value of $t_{\beta} c_{\beta-\alpha}$.

This analysis relies on our approximations of corrections to the $\lambda_{4,5,7}$ couplings; the expressions for $\delta \lambda_{4}$ and $\delta \lambda_{5}$ are verified against the mass splitting $m_{H^{ \pm}}^{2}-m_{A}^{2}$ computed by NMSSMToOLS for large $\xi_{F}$ in Fig. 9. We find very good agreement between the actual splitting from our data and the values calculated using the approximations given in Eq. (37).

We calculate the $\sigma\left(p p \rightarrow b \bar{b} A_{1}\right)$ production cross section by scaling the SM cross section by the square of the scaling of the $A_{1}$ and $b$ coupling relative to the SM value, which is provided by NMSSMTools. The SM cross section scales downward with the Higgs mass, and we fit this dependence by using the SM values provided by the 


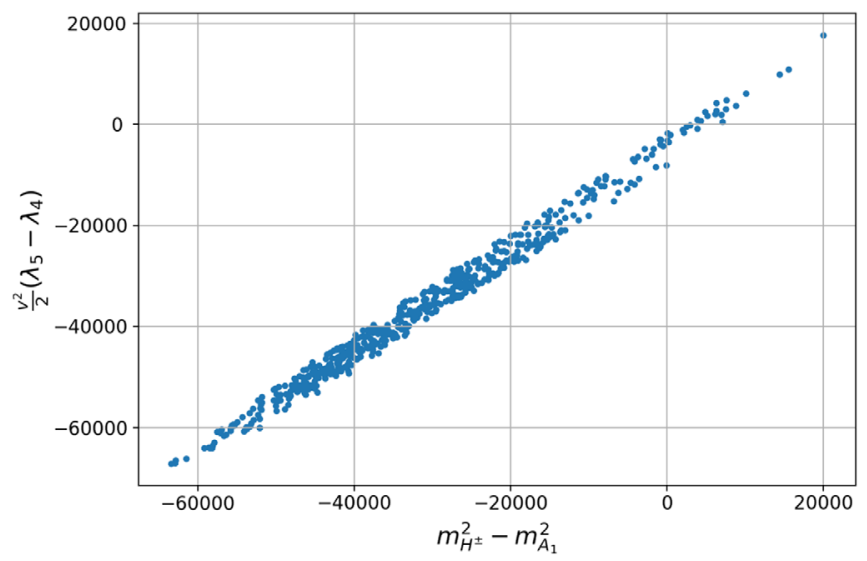

FIG. 9. Plot showing the $H^{ \pm}$and $A_{1}$ mass split calculated using our derived expression for $\frac{v^{2}}{2}\left(\lambda_{5}-\lambda_{4}\right)$ against the actual mass split for models with sizable $\xi_{F}$. There is good agreement between the two values.

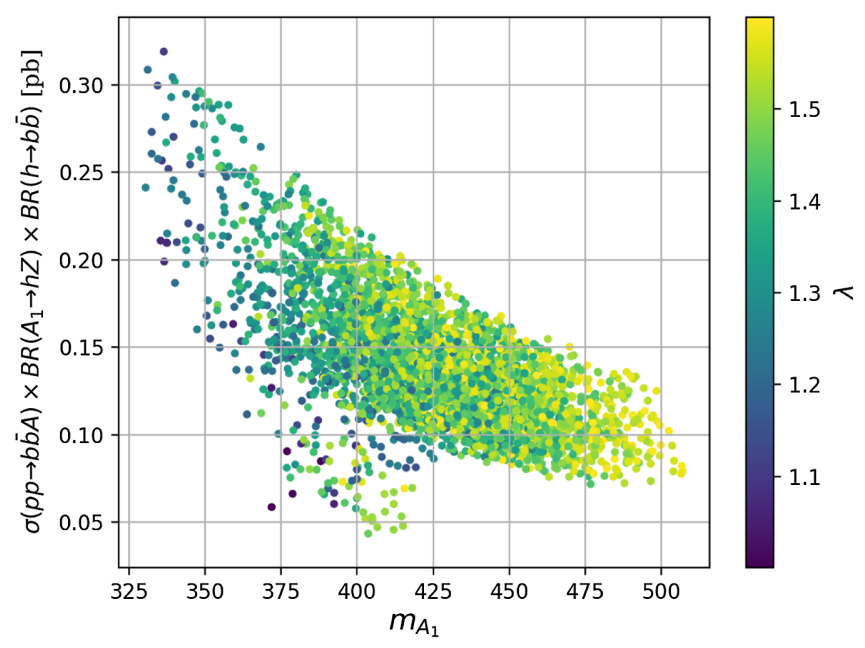

FIG. 10. Predicted value of $\sigma\left(p p \rightarrow b \bar{b} A_{1} \rightarrow h Z\right) \times \mathrm{BR}(h \rightarrow b \bar{b})$ plotted against the mass of the pseudoscalar. The value of lambda for each model is given by the colorbar. We note that one may have a production rate near $0.2 \mathrm{pb}$ for $m_{A_{1}} \approx 400 \mathrm{GeV}$.

Higgs working group [77]. The calculated value for $\sigma\left(p p \rightarrow b \bar{b} A_{1}\right)$ ranges from 300 to $1600 \mathrm{fb}$ for our particular models, with most falling within the range of 400-800 fb. With these cross section values, we find $\sigma\left(p p \rightarrow b \bar{b} A_{1}\right) \times \mathrm{BR}\left(A_{1} \rightarrow h Z\right) \times \mathrm{BR}(h \rightarrow b \bar{b})$ between
0.05 and $0.30 \mathrm{pb}$. A plot of the predicted rate against the mass of the pseudoscalar is shown in Fig. 10. We find that these models can approximately produce the observed excess at around $400 \mathrm{GeV}$, which is currently measured as $\sigma(p p \rightarrow b \bar{b} A) \times \mathrm{BR}(A \rightarrow h Z) \times \mathrm{BR}(h \rightarrow b \bar{b}) \approx$ $0.2 \mathrm{pb}$ [74]. Relevant parameter values which have been changed from the models discussed in the previous section are given by $A_{\lambda} \in[-3500,-2000] \mathrm{GeV}$, $\xi_{S} \in\left[2.5 \times 10^{9}, 1.6 \times 10^{10}\right] \mathrm{GeV}^{3}, \mu \in[-900,-500] \mathrm{GeV}$, $t_{\beta} \in[8,11], \quad \lambda \in[1.0,1.6], \quad \kappa \in[0.2,1.0], \quad$ and $\quad M_{A} \in$ $[400,410] \mathrm{GeV}$. Table II shows typical parameter values which give a rate for the pseudoscalar production near 0.2 with $m_{A_{1}}$ near $400 \mathrm{GeV}$.

With an enhanced $A_{1} \rightarrow h Z$ decay, one also expects a corresponding enhancement to the related decay $H \rightarrow h h$. CMS has updated limits on the production of a spin- 0 particle produced via gluon fusion and subsequently decaying into $h h \rightarrow b b \bar{b} \bar{b}$ [78], and one should check that this enhanced $H \rightarrow h h$ process does not exceed these limits. Indeed, the branching ratio $\operatorname{BR}(H \rightarrow h h)$ ranges mainly between about 0.5 to 0.8 in these models. However, the production rate of $H$ via gluon fusion is suppressed due to relative signs of the $H$ coupling with the top quark and the bottom quark. Within our models, $s_{\alpha} \simeq c_{\beta}$ and $c_{\alpha} \simeq s_{\beta} \simeq 1$, which differs from the case with $c_{\beta-\alpha}=0$, where instead $s_{\alpha} \simeq-c_{\beta}$. In our case, then, the coupling of the heavy Higgs to the top-quark relative to the SM value is given by $\frac{s_{\alpha}}{s_{\beta}}=\frac{1}{t_{\beta}}$ as opposed to $\frac{-1}{t_{\beta}}$ in the alignment limit. Because the gluon fusion production cross section depends on top and bottom loop contributions, such a change of sign impacts the production rate of $H$ through gluon fusion. In our models, the calculated production rate $\sigma(p p \rightarrow H \rightarrow$ $h h \rightarrow b b \bar{b} \bar{b})$ falls below the limits given by CMS. Figure 11 shows the production rate for this process against $m_{H}$ for each model. Similar conclusions apply to the $H \rightarrow Z Z$ channel.

\section{B. Precision electroweak measurements}

In the regime of large $c_{\beta-\alpha}$, precision electroweak measurements become a relevant constraint on the parameter space. We therefore calculate the values of the parameters $T$ and $S$ within our models to compare with experimental bounds. Since the singlets are heavy, we can

TABLE II. Benchmark scenarios for $b \bar{b}$-associated production of $A_{1}$ decaying into $h Z$. The column "Rate" represents the quantity $\sigma\left(p p \rightarrow b \bar{b} A_{1} \rightarrow h Z\right) \times \mathrm{BR}(h \rightarrow b \bar{b})$. All masses are given in $\mathrm{GeV}$.

\begin{tabular}{|c|c|c|c|c|c|c|c|c|c|c|c|c|c|c|}
\hline No. & $t_{\beta}$ & $\mu$ & $\lambda$ & $\kappa$ & $\xi_{S}$ & $A_{\lambda}$ & $\xi_{F}$ & $\kappa_{b}$ & $m_{h}$ & $m_{H}$ & $m_{H^{ \pm}}$ & $m_{S}$ & $m_{A_{1}}$ & Rate \\
\hline 1 & 9.6 & -587 & 1.39 & 0.326 & $3.0 \times 10^{9}$ & -2779 & $-1.2 \times 10^{6}$ & -1.11 & 124.6 & 359 & 384 & 2670 & 396 & 0.19 \\
\hline 2 & 9.2 & -579 & 1.33 & 0.500 & $2.6 \times 10^{9}$ & -3157 & $-1.5 \times 10^{6}$ & -1.22 & 125.1 & 334 & 411 & 2470 & 414 & 0.19 \\
\hline 3 & 10.5 & -576 & 1.54 & 0.328 & $2.9 \times 10^{9}$ & -2140 & $-0.8 \times 10^{6}$ & -1.15 & 123.0 & 398 & 378 & 2747 & 421 & 0.18 \\
\hline 4 & 8.0 & -784 & 1.45 & 0.405 & $5.9 \times 10^{9}$ & -3321 & $-1.9 \times 10^{6}$ & -1.18 & 123.3 & 351 & 372 & 3325 & 397 & 0.21 \\
\hline 5 & 10.8 & -586 & 1.28 & 0.464 & $3.0 \times 10^{9}$ & -3345 & $-1.6 \times 10^{6}$ & -1.21 & 122.3 & 355 & 426 & 2583 & 424 & 0.18 \\
\hline
\end{tabular}




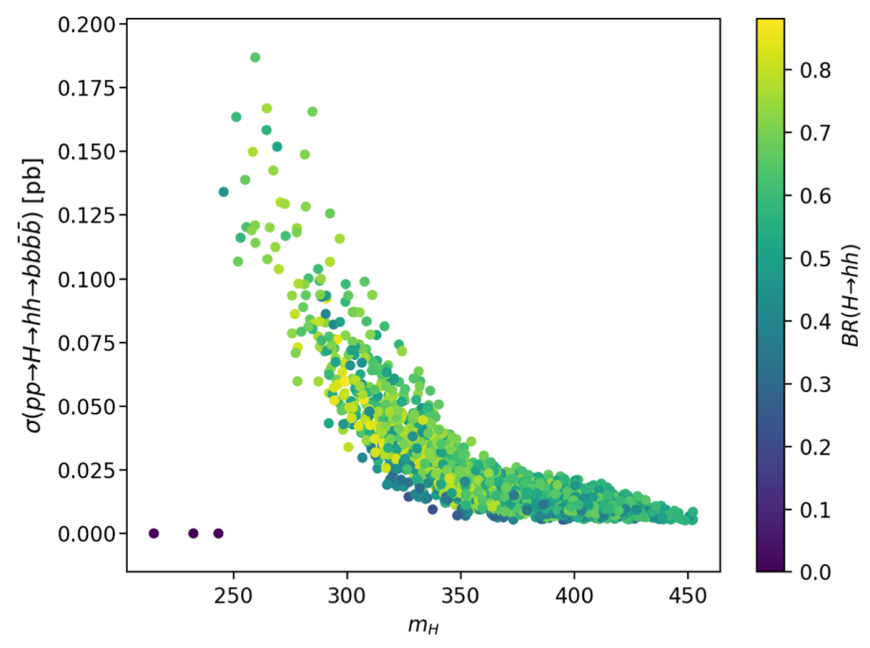

FIG. 11. Predicted rate of $H$ production through gluon fusion, decaying into $h h$ and subsequently into $b b \bar{b} \bar{b}$, against the mass of the heavy $C P$-even Higgs. The colorbar shows the branching ratio of $H \rightarrow h h$, which is enhanced in these models. The production rates fall below the current experimental upper limits from CMS.

compute the precision measurement observables within the low energy $2 \mathrm{HDM}$ effective theory. The expression for $\Delta T$ is given by [79-81]

$$
\begin{aligned}
\Delta T= & \frac{1}{16 \pi s_{W}^{2} m_{W}^{2}}\left(c _ { \beta - \alpha } ^ { 2 } \left[f\left(m_{A}, m_{H^{ \pm}}\right)\right.\right. \\
& \left.+f\left(m_{H^{ \pm}}, m_{h}\right)-f\left(m_{A}, m_{h}\right)\right] \\
& \left.+s_{\beta-\alpha}^{2}\left[f\left(m_{A}, m_{H^{ \pm}}\right)+f\left(m_{H^{ \pm}}, m_{H}\right)-f\left(m_{A}, m_{H}\right)\right]\right) \\
& +c_{\beta-\alpha}^{2} \Delta T_{\mathrm{SM}}\left(m_{H}\right)+s_{\beta-\alpha}^{2} \Delta T_{\mathrm{SM}}\left(m_{h}\right)-\Delta T_{\mathrm{SM}}\left(m_{h}\right),
\end{aligned}
$$

where $s_{W}=\sin \left(\theta_{W}\right)$ and

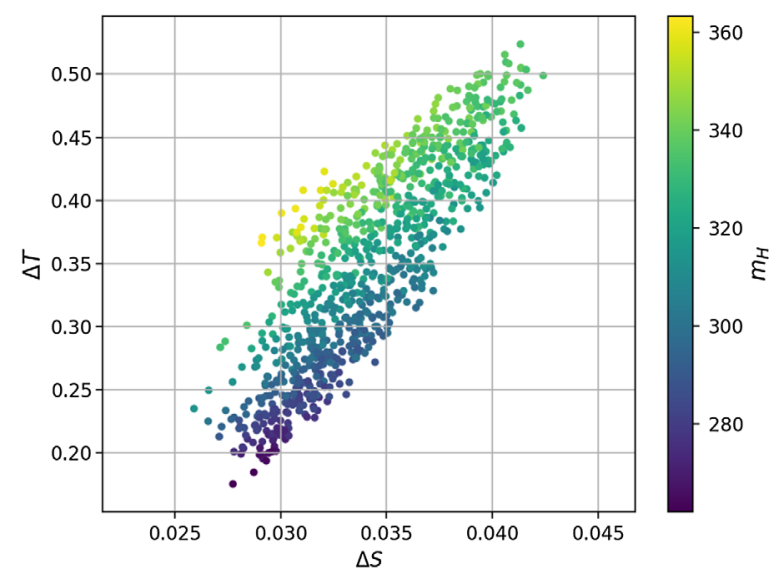

$$
\begin{gathered}
f(x, y)=\frac{x^{2}+y^{2}}{2}-\frac{x^{2} y^{2}}{x^{2}-y^{2}} \log \frac{x^{2}}{y^{2}} \\
\Delta T_{\mathrm{SM}}(m)=\frac{3}{16 \pi s_{W}^{2} m_{W}^{2}}\left[f\left(m, m_{Z}\right)-f\left(m, m_{W}\right)\right]-\frac{1}{8 \pi c_{W}^{2}}
\end{gathered}
$$

while $\Delta S$ is given by [79-81]

$$
\begin{aligned}
\Delta S= & \frac{1}{12 \pi}\left(c _ { \beta - \alpha } ^ { 2 } \left[\log \frac{m_{H}^{2}}{m_{H^{\mathrm{SM}}}^{2}}+\log \frac{m_{h} m_{A}}{m_{H^{ \pm}}^{2}}+2 \frac{m_{h}^{2} m_{A}^{2}}{\left(m_{h}^{2}-m_{A}^{2}\right)^{2}}\right.\right. \\
& \left.+\frac{\left(m_{h}^{2}+m_{A}^{2}\right)\left(m_{h}^{4}+m_{A}^{4}-4 m_{h}^{2} m_{A}^{2}\right)}{\left(m_{h}^{2}-m_{A}^{2}\right)^{3}} \log \frac{m_{h}}{m_{A}}\right] \\
& \left.+s_{\beta-\alpha}^{2}\left[\left(m_{h} \leftrightarrow m_{H}\right)\right]-\frac{5}{6}\right) .
\end{aligned}
$$

Note that due to the custodial symmetry properties, for low splitting between $m_{A}$ and $m_{H^{ \pm}}$, the terms $f\left(m_{H^{ \pm}}, m_{h, H}\right)$ and $f\left(m_{A}, m_{h, H}\right)$ in $\Delta T$ will approximately cancel; for larger splitting between the masses, i.e., lower $m_{H^{ \pm}}$, these terms have a larger contribution. The effects of these variations can be seen in Fig. 12. On the left-hand side is a plot of $\Delta T$ versus $\Delta S$ for $\xi_{F}=0$; on the right-hand side is the same plot for models with $\xi_{F} \neq 0$. In the $\xi_{F} \neq 0$ case, the splitting between $m_{H^{ \pm}}$and $m_{A}$ is reduced, as discussed in Sec. III C. In this case, we see low values of $\Delta T$. The lefthand plot also shows the dependence of $\Delta T$ on $m_{H}$ in the $\xi_{F}=0$ case, with larger values of $m_{H}$ leading to increased values of $\Delta T$.

In both cases, the value of $\Delta S$ is within the experimental limits. However, for the models presented in Sec. IV, the value of $\Delta T$ exceeds the experimental limits for a number of points. In particular, for the range of $\Delta S \approx 0.035$, the upper limit on $\Delta T$ at $99 \%$ C.L. is approximately 0.3 [82]. For $\xi_{F}=0$, one may avoid these constraints by

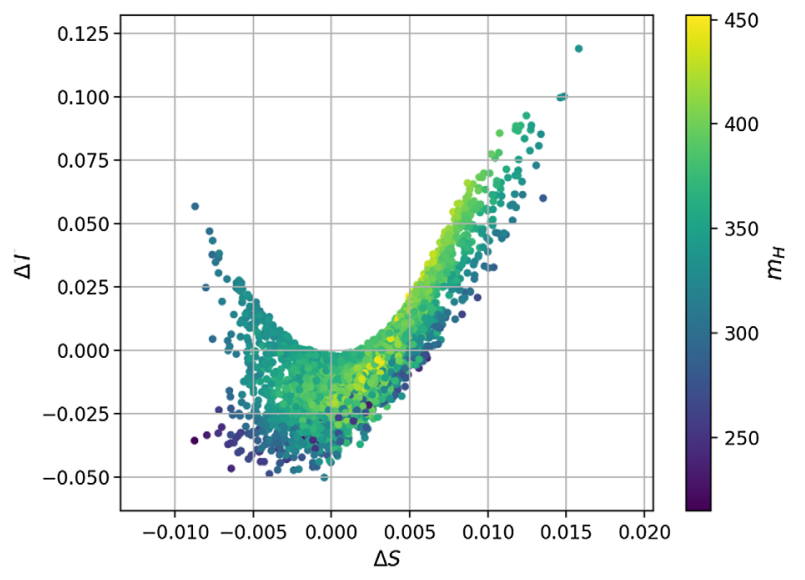

FIG. 12. Plots of the precision electroweak parameters $\Delta T$ and $\Delta S$ for the models discussed in Sec. IV (left), with $\xi_{F}=0$, and models with $\xi_{F} \neq 0$ (right). One sees much smaller magnitudes of $\Delta T$ for models with nonzero $\xi_{F}$. For large negative values of $\xi_{F}$, as in the models displayed on the right, the value of $\Delta T$ is well within experimental limits. 

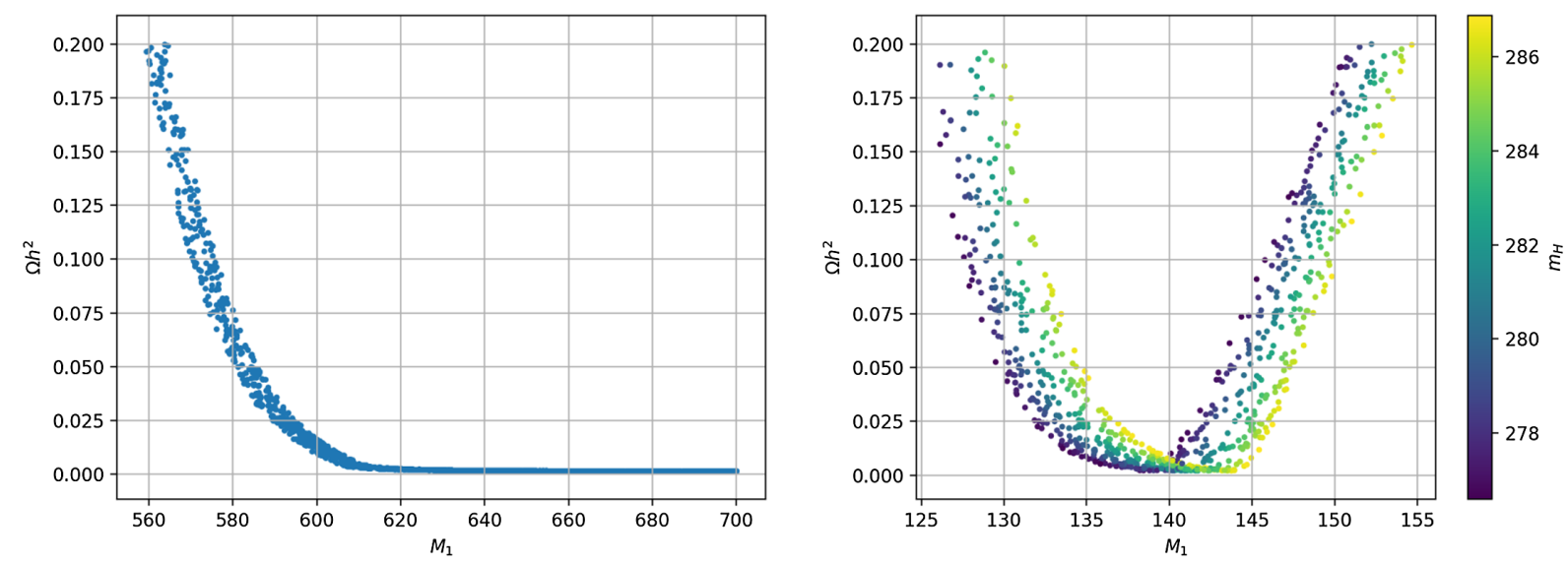

FIG. 13. Plots showing the dependence of $\Omega h^{2}$ on the value of $M_{1}$ for a single model which gives $\kappa_{b}=-1$. The left-hand plot shows the region where $M_{1} \approx m_{\tilde{S}}$, which for this model is about $600 \mathrm{GeV}$; we see the expected increase in $\Omega h^{2}$ when $M_{1} \lesssim m_{\tilde{S}}$ due to the lightest neutralino becoming primarily bino-like. The right-hand plot shows the region for which $M_{1} \approx m_{H} / 2$, where we see the expected two solutions and strong suppression when $M_{1}$ is about $140 \mathrm{GeV}$. The widening of the shape of the plots is due to scanning $\mu$ within a range of $10 \mathrm{GeV}$, which alters the value of $m_{H}$ by a few $\mathrm{GeV}$.

constraining the parameter space to lower $m_{H}$, i.e., $m_{H} \lesssim 320 \mathrm{GeV}$; an examination of Fig. 4 shows that this corresponds to $\lambda \lesssim 1.5$. One may also clearly satisfy these constraints by including a nonzero value of $\xi_{F}$. In light of these results, we conclude that while precision electroweak measurements do provide relevant constraints on the allowed parameter space for the models in Sec. IV, there are a number of existing points which agree with these constraints, and there is additionally a larger class of models which are in good agreement with measurements.

\section{DARK MATTER DENSITY AND DIRECT INTERACTION CROSS SECTION}

The question of dark matter in the NMSSM has been investigated by several authors [83-89]. In our analysis we have kept the gaugino masses and the Higgsino mass parameter $\mu$ at the $\mathrm{TeV}$ scale, implying that, provided $|\kappa|<\lambda / 2$, the lightest neutralino is mostly a singlino with mass

$$
m_{\tilde{S}} \simeq 2\left|\frac{\kappa \mu}{\lambda}\right| .
$$

As seen in Fig. 3, this condition is fulfilled in most of the parameter space we explored in this article. Such a singlino tends to mix with the Higgsino in a relevant way and, due to the large size of the couplngs $\lambda$ and $\kappa$ governing its interactions with the Higgs sector, the relic density tends to be too small to be consistent with the experimentally observed one.

Since the relic density could have a different origin from the one associated with the lightest neutralino, a small neutralino component does not lead to any phenomenological problem. However, it is easy to obtain the observed relic density by modifying the mass parameters and without affecting the Higgs phenomenology. This may be achieved, for instance, by lowering the value of the hypercharge gaugino mass $M_{1}$. For low enough values of $M_{1}$ the lightest neutralino would be bino-like and the observed relic density could be reproduced under two circumstances:

(i) values of $M_{1}$ close to a half of the lightest nonstandard Higgs masses, $m_{H} / 2$ or $m_{A_{1}} / 2$ [90,91], for which resonant annihilation could take place,

(ii) values of $M_{1}$ close to but lower than $m_{\tilde{S}}$, the socalled well-tempered singlino-bino region [92].

If either of those conditions were fulfilled, not only could the relic density be brought to agreement with the experimentally observed value, but also the spin-independent and spin-dependent interaction cross section with nuclei will be small enough to be in agreement with the current experimental constraints. In our scans, we have modified the values of $M_{1}$ and verified that this is indeed the case. In particular, for the values of the parameters present in the benchmark model 4 in Table I, Fig. 13 shows the value of $\Omega h^{2}$ for these two regions of $M_{1}$. For this point, the singlino mass is approximately $600 \mathrm{GeV}$, while the mass of the heavy $C P$-even Higgs is about $280 \mathrm{GeV}$. The widening of the shape of the plots is due to scanning $\mu$ within a range of $10 \mathrm{GeV}$, which alters the value of $m_{H}$ by a few GeV. It is clear that as the value of $M_{1}$ falls below $m_{\tilde{S}}$ and therefore the lightest neutralino becomes primarily bino-like, the relic density increases. On the other hand, for $M_{1}$ near $m_{H} / 2$, we see the two regions with $\Omega h^{2} \approx 0.1$ on either side of $m_{H} / 2 \simeq 140 \mathrm{GeV}$, where the relic density is suppressed by the resonant annihilation.

\section{CONCLUSIONS}

The current uncertainties in the determination of the Higgs coupling to bottom quarks leave room for a change 
of magnitude and sign of this coupling. In this article we have studied the possible implementation of this idea within the MSSM and the NMSSM. We have shown that in the MSSM this could only be achieved for values of $m_{A}$ and $t_{\beta}$ that are ruled out by current searches for heavy Higgs bosons decaying into tau pairs. On the other hand, in the NMSSM, consistent solutions that avoid current experimental limits may be found, but for values of the couplings $\lambda$ and $\kappa$ that lead to a Landau pole at scales below the Planck scale. This perturbativity problem may be solved by either assuming a composite Higgs model or by the introduction of an extended gauge sector that slows down the evolution of $\lambda$ at high energies.

The change of sign of the bottom coupling leads to a modification of the loop-induced couplings of the SM-like Higgs to photons and gluons that may be tested at higher luminosities at the LHC. In particular, it leads to an enhancement of the order of 20 to 25 percent of the Higgs production in the gluon fusion mode and a reduction of order of 5 to 10 percent of the width of the decay of Higgs into two photons. The modification of the gluon fusion production cross section leads already to an enhancement of the Higgs-induced diboson production cross section, which will allow one to put constraints on the allowed parameter space of the theory.

The modification of the sign of the bottom coupling also leads to a large enhancement of the radiative decay of the SM-Higgs into photons and the $\Upsilon$ meson. While such an enhancement leads to a sizable number of radiative decay events at the high luminosity LHC, the efficiency of the current searches has to be improved in order to lead to an observable signal.

In this work, we have added tadpole terms to the singlet fields that allow us to raise the value of the scalar singlets and obtain a realistic scalar spectrum. When only a tadpole for the scalar term is included, the required low values of $m_{A}$ and large values of $\lambda$ tend to lead to a charged Higgs boson mass that is lower than the top quark mass, and hence such models are strongly constrained by searches for charged Higgs bosons proceeding from the decay of top quarks. Models that avoid these constraints have masses of the charged Higgs within 10 to $15 \mathrm{GeV}$ of the top quark mass. In these models the second lightest $C P$-even and the lightest $C P$-odd scalars, which have mainly doublet components, tend to decay strongly into $H^{ \pm} W^{\mp}$, which provides an interesting search channel.

On the other hand, when a tadpole term is also included in the superpotential, the splitting between the $C P$-odd and the charged Higgs boson masses may be reduced, suppressing the decay rate of the neutral scalars into charged boson states. In this case, the decay modes $A_{1} \rightarrow h Z$ and $H \rightarrow h h$ are strongly enhanced. In particular, for values of $m_{A_{1}}$ of order of $400 \mathrm{GeV}$, which are naturally obtained within these models, the production mode $p p \rightarrow b \bar{b} A_{1} \rightarrow$ $b \bar{b} h Z$ may be sizable and can lead to an explanation of an apparent excess of $h Z$ events at the ATLAS experiment without being in conflict with the current bounds on $H \rightarrow h h$ production.

Models with light charged Higgs masses are constrained by flavor and precision measurement constraints. While the flavor constraints may be avoided by suitable supersymmetric contributions, the precision measurement constraints set a limit on the possible splittings of the charged and neutral Higgs bosons. Finally, the observed dark matter relic density may be obtained by suitable choice of the gaugino mass parameter $M_{1}$, without affecting the Higgs phenomenology.

\section{ACKNOWLEDGMENTS}

We would like to thank the Aspen Center for Physics, which is supported by the National Science Foundation under Grant No. PHYS-1066293, and where part of this work has been performed. Work at A. N. L. is supported in part by the U.S. Department of Energy under Contract No. DE-AC02-06CH11357. Work at E. F. I. is supported by the U.S. Department of Energy under Contract No. DEFG02-13ER41958.

\section{APPENDIX: RENORMALIZATION GROUP EVOLUTION}

The discussions in previous sections demonstrated that to reverse the sign of the coupling of the Higgs boson to bottom quarks, the $\lambda$ or $\kappa$ couplings need to take sizable values. However, this region of parameters leads to a Landau-pole problem, i.e., coupling constants will reach nonperturbative values at energies much lower than the GUT scale during the renormalization group evolution (RGE) [93]. This problem can be solved in two ways: the first is assuming that the rise of the quartic coupling $\lambda$ is the evidence of being in the presence of a fat Higgs model [94], namely a reflection of the composite nature of the Higgs fields, which are just mesons of a confining theory in the UV. The second is by extending the gauge groups, for example, to $S U(3)_{c} \times S U(2)_{1} \times S U(2)_{2} \times U(1)_{Y}$. More specifically, one can take the third generation and Higgs sector to be charged under $S U(2)_{1}$ while the first two generations are charged under $S U(2)_{2}$ [95]. The symmetry breaking from $S U(2)_{1} \times S U(2)_{2}$ to the regular $S U(2)$ is achieved by a bidoublet chiral field $\Sigma$ at energies $\langle\Sigma\rangle=u$ of the order of a few TeV. Large values of the $S U(2)_{1}$ coupling would allow the $\lambda$ coupling to be perturbative up to the GUT scale.

While the composite nature of the Higgs fields would be an interesting possibility, which also leads naturally to tadpole contributions to the singlet field $S$, we shall present an analysis of to what extent the model can be rendered consistent with perturbation theory up to high energy scales by the addition of extra gauge interactions. In order to get a quantitative understanding of the possible modifications of 
the RGE of the coupling $\lambda$, we performed a one-loop analysis of the evolution of the couplings to high energies. Taking the new symmetry breaking sector into consideration, above the symmetry breaking scale, and assuming just the minimal Higgs and gauge particle content to ensure the realization of this model together with approximate unification of the diagonal $S U(2)_{1} \times S U(2)_{2}, S U(3)_{c}$ and $U(1)_{Y}$ couplings at the GUT scale, the RGE equations are given by [95]

$$
\begin{aligned}
& \frac{d \tilde{\alpha}_{1}}{d t}=\frac{38}{5} \tilde{\alpha}_{1}^{2}, \\
& \frac{d \tilde{\alpha}_{2}^{(1)}}{d t}=-2\left(\tilde{\alpha}_{2}^{(1)}\right)^{2}, \\
& \frac{d \tilde{\alpha}_{2}^{(2)}}{d t}=4\left(\tilde{\alpha}_{2}^{(2)}\right)^{2}, \\
& \frac{d \tilde{\alpha}_{3}}{d t}=-2 \tilde{\alpha}_{3}^{2}, \\
& \frac{d Y_{t}}{d t}=Y_{t}\left(Y_{\lambda}+6 Y_{t}-\frac{16}{3} \tilde{\alpha}_{3}-3 \tilde{\alpha}_{2}^{(1)}-\frac{13}{15} \tilde{\alpha}_{1}\right), \\
& \frac{d Y_{\lambda}}{d t}=Y_{\lambda}\left(4 Y_{\lambda}+2 Y_{\kappa}+3 Y_{t}-3 \tilde{\alpha}_{2}^{(1)}-\frac{3}{5} \tilde{\alpha}_{1}\right), \\
& \frac{d Y_{\kappa}}{d t}=6 Y_{\kappa}\left(Y_{\lambda}+Y_{\kappa}\right),
\end{aligned}
$$

where

$$
\begin{aligned}
\tilde{\alpha}_{i}(t) & =g_{i}^{2}(t) /(4 \pi)^{2}, \\
Y_{\lambda}(t) & =\lambda^{2}(t) /(4 \pi)^{2}, \\
Y_{\kappa}(t) & =\kappa^{2}(t) /(4 \pi)^{2},
\end{aligned}
$$

$t=\ln \left(Q^{2}\right)$. In the above, $g_{i}^{(1,2)}$ correspond to the couplings of the two $S U(2)$ gauge interactions.

On the other hand, after gauge symmetry breaking, $S U(2)_{1} \times S U(2)_{2} \rightarrow S U(2)_{L}$ one is naturally left with an effective theory with the same particle content as in the NMSSM. Considering only the particles in the NMSSM, we get the following one-loop RGE equations for $\alpha$ 's and Yukawa couplings,

$$
\begin{gathered}
\frac{d \tilde{\alpha}_{1}}{d t}=\frac{33}{5} \tilde{\alpha}_{1}^{2}, \\
\frac{d \tilde{\alpha}_{2}}{d t}=\tilde{\alpha}_{2}^{2}, \\
\frac{d \tilde{\alpha}_{3}}{d t}=-3 \tilde{\alpha}_{3}^{2},
\end{gathered}
$$

$$
\begin{gathered}
\frac{d Y_{t}}{d t}=Y_{t}\left(Y_{\lambda}+6 Y_{t}-\frac{16}{3} \tilde{\alpha}_{3}-3 \tilde{\alpha}_{2}-\frac{13}{15} \tilde{\alpha}_{1}\right), \\
\frac{d Y_{\lambda}}{d t}=Y_{\lambda}\left(4 Y_{\lambda}+2 Y_{\kappa}+3 Y_{t}-3 \tilde{\alpha}_{2}-\frac{3}{5} \tilde{\alpha}_{1}\right), \\
\frac{d Y_{\kappa}}{d t}=6 Y_{\kappa}\left(Y_{\lambda}+Y_{\kappa}\right) .
\end{gathered}
$$

The modification to the renormalization group equations gives us more flexibility in the choice of $\lambda$ and $\kappa$. In Fig. 14, we display the RGE result with and without new gauge couplings, plotted along with the successful points for $\kappa_{b}=-1$ found in Sec. IV. We can see that without new gauge couplings, the constraint from the requirement of avoiding the Landau-pole problem is quite stringent since the maximum viable value for $\lambda$ is of order 0.7 and becomes smaller for larger values of $\kappa$. Therefore, all solutions with negative bottom Yukawa couplings, which are associated with values of $\lambda>1.0$, lead to the loss of perturbativity below the GUT scale. However, it is clear from the RGE equations above that large values of $\alpha_{2}^{(1)}$ lead to a smaller $\beta$ function for $\lambda$ and hence to a slower increase of $\lambda$ at large energies. This behavior is reflected in the plot, with lines of larger $g_{1}$ including a larger range of $\lambda$ and $\kappa$. Models with small $\kappa$ and $\lambda<1.5$ may remain perturbative consistent up to scales of order of $10^{16} \mathrm{GeV}$. As shown in Sec. VI B, this range of $\lambda$ 's is also preferred for consistency with precision electroweak measurements for $\xi_{F}=0$.

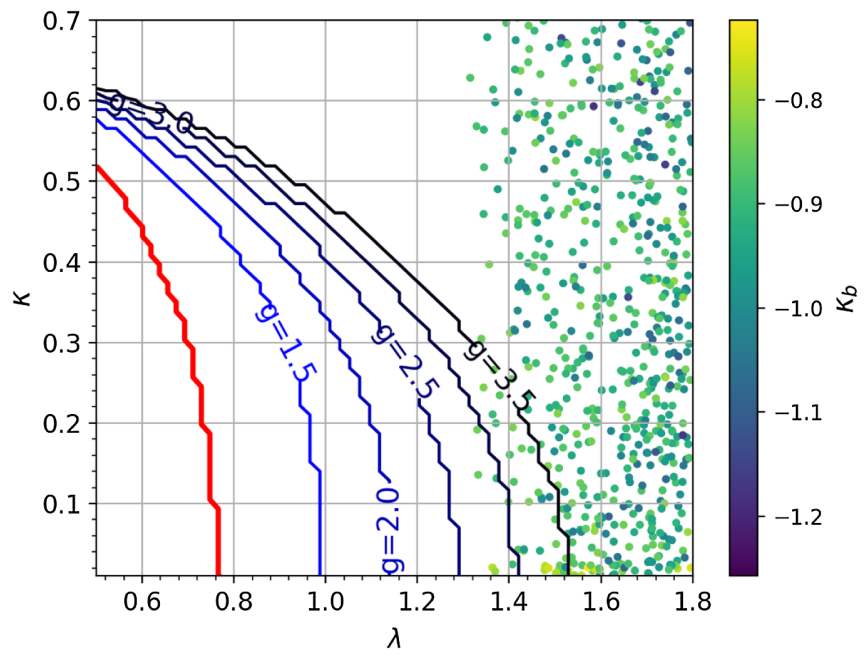

FIG. 14. Plot illustrating the Landau pole constraints on $\lambda$ and $\kappa$. Below the red line is the allowed region of $\lambda$ and $\kappa$ in the NMSSM model; the blue contours show the allowed boundaries of $\lambda$ and $\kappa$ for different values of the new $S U(2)$ coupling $g_{1}$ at $u=3 \mathrm{TeV}$. Below each line all couplings are perturbative during one-loop $\mathrm{RG}$ evolution up to energies of $10^{16} \mathrm{GeV}$. The contour lines correspond to $g_{1}=1.5,2.0,2.5,3.0$, and 3.5 from left to right. The points show the values of $\kappa$ and $\lambda$ associated with negative values of the bottom Yukawa, shown in Fig. 3. 

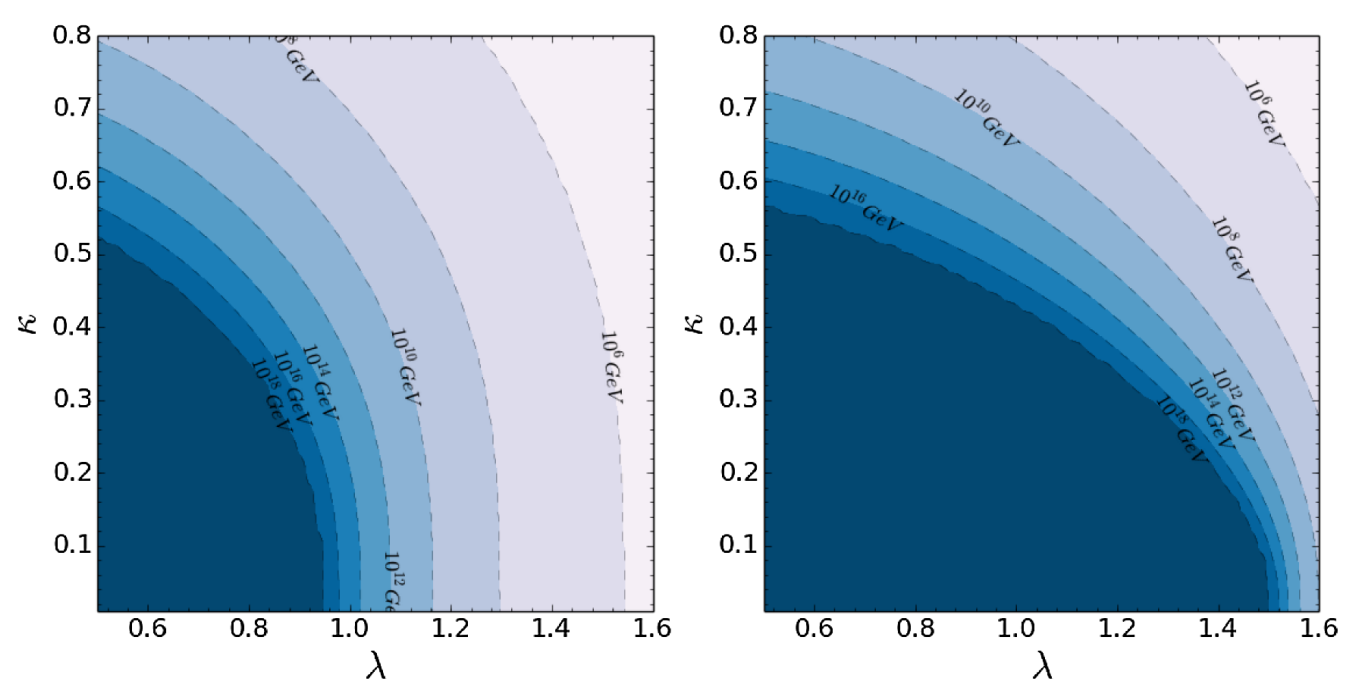

FIG. 15. Energy at which some coupling becomes nonperturbative for each $\lambda$ and $\kappa$ combination for a fixed value of $g_{1}$ at $u=3$ TeV. The left panel corresponds to $g_{1}=1.5$ while the right panel corresponds to $g_{1}=3.5$. The lines labeled with $10^{16} \mathrm{GeV}$ in the two panels are consistent with the two contour lines in Fig. 14 with the corresponding $g_{1}$ values.

In Fig. 15 we show the scale at which the coupling $\lambda$ becomes nonperturbative for different weak scale values of $\lambda$ and $\kappa$, assuming a symmetry breaking scale $u=3 \mathrm{TeV}$, for two different values of $g_{1}$ at the scale $u$, namely $g_{1}=1.5$ and $g_{1}=3.5$. It is clear that, while for $g_{1}=1.5$ perturbative consistency tends to be lost at scales of the order of $10^{6} \mathrm{GeV}$, for $g_{1}=3.5$ a wide range of models leading to the inversion of the Higgs coupling to bottom quarks are perturbative consistent up to scales of the order of $10^{10} \mathrm{GeV}$.
[1] G. Aad et al. (ATLAS Collaboration), Observation of a new particle in the search for the Standard Model Higgs boson with the ATLAS detector at the LHC, Phys. Lett. B 716, 1 (2012); Measurements of Higgs boson production and couplings in diboson final states with the ATLAS detector at the LHC, Phys. Lett. B 726, 88 (2013).

[2] S. Chatrchyan et al. (CMS Collaboration), Observation of a new boson at a mass of $125 \mathrm{GeV}$ with the CMS experiment at the LHC, Phys. Lett. B 716, 30 (2012); Observation of a new boson with mass near $125 \mathrm{GeV}$ in pp collisions at $\sqrt{s}=7$ and $8 \mathrm{TeV}$, J. High Energy Phys. 06 (2013) 081.

[3] G. Aad et al. (ATLAS and CMS Collaborations), Measurements of the Higgs boson production and decay rates and constraints on its couplings from a combined ATLAS and CMS analysis of the LHC pp collision data at $\sqrt{s}=7$ and 8 TeV, J. High Energy Phys. 08 (2016) 045.

[4] M. Aaboud et al. (ATLAS Collaboration), Evidence for the associated production of the Higgs boson and a top quark pair with the ATLAS detector, Phys. Rev. D 97, 072003 (2018).

[5] CMS Collaboration, Search for Higgs boson production in association with top quarks in multilepton final states at $\sqrt{s}=13 \mathrm{TeV}$, Report No. CMS-PAS-HIG-17-004.
[6] M. Aaboud et al. (ATLAS Collaboration), Evidence for the $H \rightarrow b \bar{b}$ decay with the ATLAS detector, J. High Energy Phys. 12 (2017) 024.

[7] A. M. Sirunyan et al. (CMS Collaboration), Evidence for the Higgs boson decay to a bottom quark-antiquark pair, Phys. Lett. B 780, 501 (2018).

[8] P. M. Ferreira, J. F. Gunion, H. E. Haber, and R. Santos, Probing wrong sign Yukawa couplings at the LHC and a future linear collider, Phys. Rev. D 89, 115003 (2014).

[9] D. Aloni, Y. Nir, and E. Stamou, Large $\operatorname{BR}(h \rightarrow \tau \mu)$ in the MSSM?, J. High Energy Phys. 04 (2016) 162.

[10] M. Bauer, M. Carena, and K. Gemmler, Flavor from the electroweak scale, J. High Energy Phys. 11 (2015) 016.

[11] K. Inoue, A. Kakuto, H. Komatsu, and S. Takeshita, Lowenergy parameters and particle masses in a supersymmetric grand unified model, Prog. Theor. Phys. 67, 1889 (1982); R. A. Flores and M. Sher, Higgs masses in the standard, multi-Higgs and supersymmetric models, Ann. Phys. (N.Y.) 148, 95 (1983).

[12] J. F. Gunion and H. E. Haber, Higgs bosons in supersymmetric models. 1., Nucl. Phys. B272, 1 (1986); Erratum, 402, 567 (1993).

[13] M. S. Carena and H. E. Haber, Higgs boson theory and phenomenology, Prog. Part. Nucl. Phys. 50, 63 (2003). 
[14] A. Djouadi, The anatomy of electroweak symmetry breaking. II. The Higgs bosons in the minimal supersymmetric model, Phys. Rep. 459, 1 (2008).

[15] U. Ellwanger, C. Hugonie, and A. M. Teixeira, The next-tominimal supersymmetric Standard Model, Phys. Rep. 496, 1 (2010).

[16] S. P. Martin, A supersymmetry primer, Adv. Ser. Dir. High Energy Phys. 21, 1 (2010); 18, 1 (1998).

[17] M. Aaboud et al. (ATLAS Collaboration), Search for additional heavy neutral Higgs and gauge bosons in the ditau final state produced in $36 \mathrm{fb}^{-1}$ of pp collisions at $\sqrt{s}=13 \mathrm{TeV}$ with the ATLAS detector, J. High Energy Phys. 01 (2018) 055.

[18] A. M. Sirunyan et al. (CMS Collaboration), Search for additional neutral MSSM Higgs bosons in the $\tau \tau$ final state in proton-proton collisions at $\sqrt{s}=13 \mathrm{TeV}$, arXiv:1803 .06553 .

[19] P. M. Ferreira, S. Liebler, and J. Wittbrodt, Wrong or right: $p p \rightarrow A \rightarrow Z h$ and the sign of the two-Higgs-doublet model, Phys. Rev. D 97, 055008 (2018).

[20] D. Das, A. Kundu, and I. Saha, Higgs data does not rule out a sequential fourth generation with an extended scalar sector, Phys. Rev. D 97, 011701 (2018).

[21] H. Georgi and D. V. Nanopoulos, Suppression of flavor changing effects from neutral spinless meson exchange in gauge theories, Phys. Lett. B 82, 95 (1979); J. F. Donoghue and L. F. Li, Properties of charged Higgs bosons, Phys. Rev. D 19, 945 (1979); L. Lavoura and J. P. Silva, Fundamental $C P$ violating quantities in a $\mathrm{SU}(2) \times \mathrm{U}(1)$ model with many Higgs doublets, Phys. Rev. D 50, 4619 (1994); L. Lavoura, Signatures of discrete symmetries in the scalar sector, Phys. Rev. D 50, 7089 (1994); F. J. Botella and J. P. Silva, Jarlskog-like invariants for theories with scalars and fermions, Phys. Rev. D 51, 3870 (1995).

[22] G. C. Branco, L. Lavoura, and J. P. Silva, CP Violation (Oxford University Press, Oxford, UK, 1999).

[23] J.F. Gunion and H.E. Haber, The $C P$ conserving twoHiggs-doublet model: The approach to the decoupling limit, Phys. Rev. D 67, 075019 (2003).

[24] N. Craig, J. Galloway, and S. Thomas, Searching for signs of the second Higgs doublet, arXiv:1305.2424.

[25] M. Carena, H. E. Haber, I. Low, N. R. Shah, and C. E. M. Wagner, Complementarity between nonstandard Higgs boson searches and precision Higgs boson measurements in the MSSM, Phys. Rev. D 91, 035003 (2015).

[26] J. Bernon, J. F. Gunion, H. E. Haber, Y. Jiang, and S. Kraml, Scrutinizing the alignment limit in two-Higgsdoublet models: $\mathrm{m}_{h}=125 \mathrm{GeV}$, Phys. Rev. D 92, 075004 (2015).

[27] J. Bernon, J. F. Gunion, H. E. Haber, Y. Jiang, and S. Kraml, Scrutinizing the alignment limit in two-Higgs-doublet models. II. $\mathrm{m}_{H}=125 \mathrm{GeV}$, Phys. Rev. D 93, 035027 (2016).

[28] H. E. Haber and R. Hempfling, The renormalization group improved Higgs sector of the minimal supersymmetric model, Phys. Rev. D 48, 4280 (1993).

[29] M. Carena, J. R. Espinosa, M. Quiros, and C. E. M. Wagner, Analytical expressions for radiatively corrected Higgs masses and couplings in the MSSM, Phys. Lett. B 355, 209 (1995).
[30] G. Lee and C.E. M. Wagner, Higgs bosons in heavy supersymmetry with an intermediate $\mathrm{m}_{A}$, Phys. Rev. D 92, 075032 (2015).

[31] N. Blinov and D. E. Morrissey, Vacuum stability and the MSSM Higgs mass, J. High Energy Phys. 03 (2014) 106.

[32] H. E. Haber and R. Hempfling, Can the Mass of the Lightest Higgs Boson of the Minimal Supersymmetric Model be Larger than m(Z)?, Phys. Rev. Lett. 66, 1815 (1991); Y. Okada, M. Yamaguchi, and T. Yanagida, Upper bound of the lightest Higgs boson mass in the minimal supersymmetric standard model, Prog. Theor. Phys. 85, 1 (1991); J. R. Ellis, G. Ridolfi, and F. Zwirner, Radiative corrections to the masses of supersymmetric Higgs bosons, Phys. Lett. B 257, 83 (1991).

[33] See e.g., M. S. Carena, H. E. Haber, S. Heinemeyer, W. Hollik, C. E. M. Wagner, and G. Weiglein, Reconciling the two loop diagrammatic and effective field theory computations of the mass of the lightest $C P$-even Higgs boson in the MSSM, Nucl. Phys. B580, 29 (2000); G. Degrassi, S. Heinemeyer, W. Hollik, P. Slavich, and G. Weiglein, Towards high precision predictions for the MSSM Higgs sector, Eur. Phys. J. C 28, 133 (2003).

[34] J. R. Espinosa and M. Quiros, On Higgs boson masses in nonminimal supersymmetric standard models, Phys. Lett. B 279, 92 (1992).

[35] E. Bagnaschi, G. F. Giudice, P. Slavich, and A. Strumia, Higgs mass and unnatural supersymmetry, J. High Energy Phys. 09 (2014) 092.

[36] T. Hahn, S. Heinemeyer, W. Hollik, H. Rzehak, and G. Weiglein, High-Precision Predictions for the Light $C P$-Even Higgs Boson Mass of the Minimal Supersymmetric Standard Model, Phys. Rev. Lett. 112, 141801 (2014).

[37] J. Pardo Vega and G. Villadoro, SusyHD: Higgs mass determination in supersymmetry, J. High Energy Phys. 07 (2015) 159.

[38] P. Draper, G. Lee, and C. E. M. Wagner, Precise estimates of the Higgs mass in heavy supersymmetry, Phys. Rev. D 89 , 055023 (2014).

[39] H. Bahl and W. Hollik, Precise prediction for the light MSSM Higgs boson mass combining effective field theory and fixedorder calculations, Eur. Phys. J. C 76, 499 (2016).

[40] C. Panagiotakopoulos and K. Tamvakis, Stabilized NMSSM without domain walls, Phys. Lett. B 446, 224 (1999).

[41] C. Panagiotakopoulos and K. Tamvakis, New minimal extension of MSSM, Phys. Lett. B 469, 145 (1999).

[42] C. Panagiotakopoulos and A. Pilaftsis, Higgs scalars in the minimal nonminimal supersymmetric standard model, Phys. Rev. D 63, 055003 (2001).

[43] See Chapter 1.3 of D. M. Asner, T. Barklow, C. Calancha, K. Fujii, N. Graf, H. E. Haber, A. Ishikawa, S. Kanemura et al., ILC Higgs White Paper, arXiv:1310.0763.

[44] H.E. Haber, The Higgs data and the decoupling limit, arXiv:1401.0152.

[45] M. S. Carena, H. E. Haber, H. E. Logan, and S. Mrenna, Distinguishing a MSSM Higgs boson from the SM Higgs boson at a linear collider, Phys. Rev. D 65, 055005 (2002); Erratum, Phys. Rev. D 65, 099902(E) (2002).

[46] M. Carena, S. Mrenna, and C. E. M. Wagner, MSSM Higgs boson phenomenology at the Tevatron collider, Phys. Rev. D 60, 075010 (1999). 
[47] R. Hempfling, Yukawa coupling unification with supersymmetric threshold corrections, Phys. Rev. D 49, 6168 (1994).

[48] L. J. Hall, R. Rattazzi, and U. Sarid, The top quark mass in supersymmetric SO(10) unification, Phys. Rev. D 50, 7048 (1994).

[49] M. Carena, M. Olechowski, S. Pokorski, and C. E. M. Wagner, Electroweak symmetry breaking and bottom-top Yukawa unification, Nucl. Phys. B426, 269 (1994).

[50] M. Carena, H. E. Haber, I. Low, N. R. Shah, and C. E. M. Wagner, Alignment limit of the NMSSM Higgs sector, Phys. Rev. D 93, 035013 (2016).

[51] U. Ellwanger, J. F. Gunion, and C. Hugonie, NMHDECAY: A Fortran code for the Higgs masses, couplings and decay widths in the NMSSM, J. High Energy Phys. 02 (2005) 066.

[52] CMS Collaboration, Search for charged Higgs bosons with the $\mathrm{H}^{+}$to tau nu decay channel in the fully hadronic final state at sqrt $\mathrm{s}=8 \mathrm{TeV}$, Report No. CMS-PAS-HIG14-020.

[53] CMS Collaboration, Combined measurements of the Higgs boson's couplings at $\sqrt{s}=13 \mathrm{TeV}$, Report No. CMS-PASHIG-17-031.

[54] ATLAS Collaboration, Combined measurements of Higgs boson production and decay in the $H \rightarrow Z Z^{?} \rightarrow 4 \ell$ and $H \rightarrow \gamma \gamma$ channels using $\sqrt{s}=13 \mathrm{TeV}$ pp collision data collected with the ATLAS experiment, Report No. ATLAS-CONF-2017-047.

[55] S. Dawson et al., Working Group Report: Higgs boson, arXiv:1310.8361.

[56] A. Djouadi, Squark effects on Higgs boson production and decay at the LHC, Phys. Lett. B 435, 101 (1998).

[57] R. Dermisek and I. Low, Probing the stop sector and the sanity of the MSSM with the Higgs boson at the LHC, Phys. Rev. D 77, 035012 (2008).

[58] M. Carena, S. Gori, N. R. Shah, and C. E. M. Wagner, A $125 \mathrm{GeV}$ SM-like Higgs in the MSSM and the $\gamma \gamma$ rate, J. High Energy Phys. 03 (2012) 014.

[59] M. Carena, S. Gori, N. R. Shah, C. E. M. Wagner, and L. T. Wang, Light stops, light staus and the $125 \mathrm{GeV}$ Higgs, J. High Energy Phys. 08 (2013) 087.

[60] T. Modak, J. C. Romao, S. Sadhukhan, J. P. Silva, and R. Srivastava, Constraining wrong sign $h b b$ couplings with $h \rightarrow \Upsilon_{\gamma}$, Phys. Rev. D 94, 075017 (2016).

[61] G. T. Bodwin, H. S. Chung, J. H. Ee, J. Lee, and F. Petriello, Relativistic corrections to Higgs boson decays to quarkonia, Phys. Rev. D 90, 113010 (2014).

[62] https://twiki.cern.ch/twiki/bin/view/LHCPhysics/LHCHX SWG.

[63] G. Aad et al. (ATLAS Collaboration), Search for Higgs and $\mathrm{Z}$ Boson Decays to $J / \psi \gamma$ and $\Upsilon(n S) \gamma$ with the ATLAS Detector, Phys. Rev. Lett. 114, 121801 (2015).

[64] R. Contino et al., Physics at a $100 \mathrm{TeV}$ pp collider: Higgs and EW symmetry breaking studies, arXiv:1606.09408.

[65] J. L. Hewett, Can $b \rightarrow s \gamma$ Close the Supersymmetric Higgs Production Window?, Phys. Rev. Lett. 70, 1045 (1993).

[66] M. Misiak and M. Steinhauser, Weak radiative decays of the B meson and bounds on $M_{H^{ \pm}}$in the two-Higgs-doublet model, Eur. Phys. J. C 77, 201 (2017).

[67] R. Barbieri and G. F. Giudice, $b \rightarrow s \gamma$ decay and supersymmetry, Phys. Lett. B 309, 86 (1993).
[68] M. Ciuchini, G. Degrassi, P. Gambino, and G. F. Giudice, Next-to-leading QCD corrections to $B \rightarrow X_{s} \gamma$ : Standard model and two-Higgs-doublet model, Nucl. Phys. B527, 21 (1998).

[69] G. Degrassi, P. Gambino, and G. F. Giudice, $B \rightarrow X(s \gamma)$ in supersymmetry: Large contributions beyond the leading order, J. High Energy Phys. 12 (2000) 009.

[70] M. Carena, D. Garcia, U. Nierste, and C. E. M. Wagner, $b \rightarrow s \gamma$ and supersymmetry with large $t_{\beta}$, Phys. Lett. B 499, 141 (2001).

[71] A. J. Buras, P.H. Chankowski, J. Rosiek, and L. Slawianowska, $\Delta M_{d, s}, B^{0} d, s \rightarrow \mu^{+} \mu^{-}$and $B \rightarrow X_{s} \gamma$ in supersymmetry at large $t_{\beta}$, Nucl. Phys. B659, 3 (2003).

[72] F. Gabbiani, E. Gabrielli, A. Masiero, and L. Silvestrini, A complete analysis of FCNC and $C P$ constraints in general SUSY extensions of the standard model, Nucl. Phys. B477, 321 (1996).

[73] M. Carena, A. Menon, and C. E. M. Wagner, Minimal flavor violation and the scale of supersymmetry breaking, Phys. Rev. D 79, 075025 (2009).

[74] ATLAS Collaboration, Search for heavy resonances decaying to a $W$ or $Z$ boson and a Higgs boson in final states with leptons and $b$-jets in $36.1 \mathrm{fb}^{-1}$ of $p p$ collision data at $\sqrt{s}=13 \mathrm{TeV}$ with the ATLAS detector, Report No. ATLAS-CONF-2017-055.

[75] S. von Buddenbrock, A. S. Cornell, A. Fadol, M. Kumar, B. Mellado, and X. Ruan, Multi-lepton signatures of additional scalar bosons beyond the Standard Model at the LHC, arXiv:1711.07874.

[76] S. von Buddenbrock, N. Chakrabarty, A. S. Cornell, D. Kar, M. Kumar, T. Mandal, B. Mellado, B. Mukhopadhyaya, R. G. Reed, and X. Ruan, Phenomenological signatures of additional scalar bosons at the LHC, Eur. Phys. J. C 76, 580 (2016).

[77] D. de Florian et al. (LHC Higgs Cross Section Working Group), Handbook of LHC Higgs cross sections: 4. Deciphering the nature of the Higgs sector, arXiv:1610.07922.

[78] CMS Collaboration, Search for resonant pair production of Higgs bosons decaying to bottom quark-antiquark pairs in proton-proton collisions at $13 \mathrm{TeV}$, Report No. CMS-PASHIG-17-009.

[79] T. Inami, C. S. Lim, and A. Yamada, Radiative correction parameter $\mathrm{S}$ in beyond the standard models, Mod. Phys. Lett. A 07, 2789 (1992).

[80] P. H. Chankowski, T. Farris, B. Grzadkowski, J. F. Gunion, J. Kalinowski, and M. Krawczyk, Do precision electroweak constraints guarantee e+e-collider discovery of at least one Higgs boson of a two-Higgs-doublet model?, Phys. Lett. B 496, 195 (2000).

[81] D. Choudhury, T. M. P. Tait, and C. E. M. Wagner, Probing heavy Higgs boson models with a TeV linear collider, Phys. Rev. D 65, 115007 (2002).

[82] C. Patrignani et al. (Particle Data Group), Review of particle physics, Chin. Phys. C 40, 100001 (2016).

[83] D. G. Cerdeno, C. Hugonie, D. E. Lopez-Fogliani, C. Munoz, and A. M. Teixeira, Theoretical predictions for the direct detection of neutralino dark matter in the NMSSM, J. High Energy Phys. 12 (2004) 048.

[84] G. Belanger, F. Boudjema, C. Hugonie, A. Pukhov, and A. Semenov, Relic density of dark matter in the NMSSM, J. Cosmol. Astropart. Phys. 09 (2005) 001. 
[85] J. F. Gunion, D. Hooper, and B. McElrath, Light neutralino dark matter in the NMSSM, Phys. Rev. D 73, 015011 (2006).

[86] D. G. Cerdeno, E. Gabrielli, D. E. Lopez-Fogliani, C. Munoz, and A. M. Teixeira, Phenomenological viability of neutralino dark matter in the NMSSM, J. Cosmol. Astropart. Phys. 06 (2007) 008.

[87] J. J. Cao, K.-i. Hikasa, W. Wang, and J. M. Yang, Light dark matter in NMSSM and implication on Higgs phenomenology, Phys. Lett. B 703, 292 (2011).

[88] J. Kozaczuk and S. Profumo, Light NMSSM neutralino dark matter in the wake of CDMS II and a $126 \mathrm{GeV}$ Higgs boson, Phys. Rev. D 89, 095012 (2014).

[89] L. Wang, R. Shi, and X. F. Han, Wrong sign Yukawa coupling of the 2HDM with a singlet scalar as dark matter confronted with dark matter and Higgs data, Phys. Rev. D 96, 115025 (2017).
[90] K. Griest and D. Seckel, Three exceptions in the calculation of relic abundances, Phys. Rev. D 43, 3191 (1991).

[91] M. Drees and M. M. Nojiri, The neutralino relic density in minimal $N=1$ supergravity, Phys. Rev. D 47, 376 (1993).

[92] S. Baum, M. Carena, N. R. Shah, and C.E. M. Wagner, Higgs portals for thermal dark matter-EFT perspectives and the NMSSM, J. High Energy Phys. 04 (2018) 069.

[93] R. B. Nevzorov and M. A. Trusov, Yad. Fiz. 64, 1589 (2001) [Renormalization of the soft SUSY breaking terms in the strong Yukawa coupling limit in the NMSSM, Phys. At. Nucl. 64, 1513 (2001)].

[94] R. Harnik, G. D. Kribs, D. T. Larson, and H. Murayama, The minimal supersymmetric fat Higgs model, Phys. Rev. D 70, 015002 (2004).

[95] P. Batra, A. Delgado, D. E. Kaplan, and T. M. P. Tait, Running into new territory in SUSY parameter space, J. High Energy Phys. 06 (2004) 032. 